\title{
A Schistosomiasis Model with Praziquantel Resistance
}

\author{
Longxing $\mathrm{Qi}^{1}$ and Jing-an $\mathrm{Cui}^{2}$ \\ ${ }^{1}$ School of Mathematical Sciences, Anhui University, Hefei 230601, China \\ ${ }^{2}$ College of Science, Beijing University of Civil Engineering and Architecture, Beijing 100044, China
}

Correspondence should be addressed to Longxing Qi; qilx@ahu.edu.cn

Received 28 August 2012; Accepted 23 October 2012

Academic Editor: Cengiz Çinar

Copyright (C) 2013 L. Qi and J.-a. Cui. This is an open access article distributed under the Creative Commons Attribution License, which permits unrestricted use, distribution, and reproduction in any medium, provided the original work is properly cited.

\begin{abstract}
A compartmental model is established for schistosomiasis with praziquantel resistance. The model considers the impact of genetic resistance and drug treatment on the transmission of schistosomiasis. We calculate the basic reproductive number and discuss the existence and stability of disease-free equilibrium, boundary equilibrium, and coexistence equilibrium. Our analysis shows that regardless of whether drug treatment leads to the emergence of resistance, once the impact of genetic resistance is larger, the resistant strain will be dominant, which is detrimental to the control of schistosomiasis. In addition, once the proportion of human with drug-resistant strain produced by drug treatment is larger, the number of human and snails with resistant strain is larger. This is not a good result for drug treatment with praziquantel.
\end{abstract}

\section{Introduction}

Currently, treatment of human beings infected by schistosomiasis primarily focuses on chemotherapy with praziquantel (PZQ). PZQ appeared as a new schistosomicidal compound during the 1970s [1]. In recent years, PZQ has become the drug of choice in most endemic areas because of its efficacy, its ease of administration, its tolerable side-effects, and its cost [1]. Although the effectiveness of PZQ against schistosomiasis is well documented, the precise mode of action of the drug has not clearly defined [2]. It is reported that the chemotherapy of many helminth infections is complicated by the occurrence of drug resistance and drug tolerance (a natural resistance) to certain anthelmintics [2]. Not surprisingly, recent epidemiological evidence suggests the emergence of PZQ-resistant \tolerant schistosomes $[1,3,4]$. Resistance is defined as a genetically transmitted loss of sensitivity in a parasite population that was previously sensitive to a given drug [2]. Tolerance is an innate insusceptibility of a parasite to a drug, with the caveat that the parasite must not have been previously exposed to the drug [2]. The first report of possible PZQ resistance came from an intensive focus in northern Senegal, where the drug had produced very low cure rates (18-39\%) [5,6]. And snails collected in the area carried schistosoma strains. When tested in the laboratory, those snails had a decreased susceptibility to PZQ $[7,8]$.
Additional PZQ-resistant evidence was collected in Egypt $[4,9]$. Preliminary studies have begun on these isolates to identify genetic, physiological, and morphological characteristics associated with PZQ resistance, and some of these may find use as markers for monitoring whether or not resistance is developing in endemic areas, where the drug is used [10].

Many papers have reported that drug treatment results in the emergence of schistosome resistance to PZQ [11-13]. Drug treatment can remove drug-susceptible parasites in infected human beings, while resistant parasites survive. However, many investigations find that traits of PZQ resistance of Schistosoma mansoni are dominant inheritance [14-16]. The resistant worms can reproduce and pass the resistant genes to the next generation. Furthermore, the resistance of Schistosoma mansoni to PZQ can be expressed in eggs, miracidia, cercariae, adults, and all stages of development [14-16]. In other words, some definitive hosts carrying resistant schistosomes can infect snails and make those snails carry resistant schistosomes. On the contrary, some snails carrying resistant schistosomes can infect definitive hosts and also make those definitive hosts carry resistant schistosomes. It goes on, the control of schistosomiasis will face enormous difficulties. Therefore, it is necessary to study the impact of this schistosome genetic resistance on the transmission of schistosomiasis. 
In previous schistosomiasis models, resistant problems have been studied by considering that the resistance of schistosomiasis is due to drug treatment [11-13]. In [11, 13], the authors proposed a multistrain schistosome model including sensitive and resistant parasite strains. Their goal was to infer the impact of drug treatment on the maintenance of schistosome genetic diversity. In their assumptions, the drugsensitive parasite strain had an additional per capita death rate, $\sigma$, due to treatment. For a parasite strain that had developed drug resistance with a resistance level $\theta(\theta>1)$, this treatment-related death rate was assumed to be reduced by the factor $\theta$ to $\sigma / \theta$. Their results implied that higher treatment rate could allow for coexistence between sensitive and resistant parasite strains. In [12], the authors formulated a deterministic model with multiple strains of schistosomes in order to explore the role of drug treatment in the maintenance of a polymorphism of parasite strains that differed in their resistance levels. And snails infected by parasite strains were divided into multistrain subclasses according to the different level $\theta_{i}$ of parasite strains. Analysis of the model showed that the likelihood that resistant strains would increase in frequency depended on the interplay between their relative fitness, the cost of resistance, and the degree of selection pressure exerted by drug treatments.

Motivated by [11-13], we establish a new model considering hosts with sensitive and resistant strains in this paper. Our purpose is mainly to study the impact of drug treatment and genetic resistance on the transmission of schistosomiasis.

Our paper is organized as follows. In Section 2, we establish a mathematical model with praziquantel resistance and obtain basic reproductive number and existence of equilibria. And then the stability of disease-free equilibrium is obtained in Section 3. Section 4 devotes to stability analysis of boundary equilibria. In Section 5, stability analysis of endemic equilibrium is performed.

\section{Mathematical Model}

According to different level of parasite strains, we divide infected hosts into sensitive and resistant strains. Considering resistance and inheritance of resistance, new resistant strains are composed of two parts. We classify definitive and intermediate hosts as susceptible, sensitive and resistant in the following:

(i) $X_{s}(t)$, the population of susceptible human;

(ii) $X_{i}(t)$, the population of human infected with sensitive parasite strain;

(iii) $X_{r}(t)$, the population of human infected with resistant parasite strain;

(iv) $Y_{s}(t)$, the population of susceptible snail host;

(v) $Y_{i}(t)$, the population of snail host carrying sensitive parasites;

(vi) $Y_{r}(t)$, the population of snail host carrying resistant parasites.

We follow some of the available models for schistosomiasis [17-19] and assume that the reproduction rate of hosts is constant, and we ignore the recovery class of host since the life span of infected is short in comparison to that of human $[18,20]$. All parameters in the model are assumed to be nonnegative constants:

(i) $\Lambda_{1}$, recruitment rate of human;

(ii) $\mu_{1}$, per capita natural death rate of human;

(iii) $\alpha_{1}$, per capita disease-induced death rate of human;

(iv) $\beta_{1}$, per capita contact transmission rate from infected snails to susceptible human;

(v) $\Lambda_{2}$, recruitment rate of snails;

(vi) $\mu_{2}$, per capita natural death rate of snails;

(vii) $\alpha_{2}$, per capita disease-induced death rate of snails;

(viii) $\beta_{2}$, per capita contact transmission rate from infected human to susceptible snails;

(ix) $\theta$, the rate of treatment for infected human;

(x) $\rho$, the proportion of human with drug-resistant strain produced by treatment;

(xi) $k$, represents the impact of inheritance and the cost of resistance on transmission rate, we assume that $k \leq 1$ since the transmission rate is reduced due to resistance [12];

(xii) $k_{1}$, represents the impact of resistance on diseaseinduced death rate of human;

(xiii) $k_{2}$, represents the impact of resistance on diseaseinduced death rate of snails.

Then, we have a model with the form:

$$
\begin{aligned}
& \frac{d X_{s}}{d t}=\Lambda_{1}-\beta_{1} X_{s} Y_{i}-k \beta_{1} X_{s} Y_{r}-\mu_{1} X_{s}+(1-\rho) \sigma X_{i} \\
& \frac{d X_{i}}{d t}=\beta_{1} X_{s} Y_{i}-\mu_{1} X_{i}-\alpha_{1} X_{i}-\sigma X_{i} \\
& \frac{d X_{r}}{d t}=k \beta_{1} X_{s} Y_{r}+\rho \sigma X_{i}-\mu_{1} X_{r}-k_{1} \alpha_{1} X_{r} \\
& \frac{d Y_{s}}{d t}=\Lambda_{2}-\beta_{2} Y_{s} X_{i}-k \beta_{2} Y_{s} X_{r}-\mu_{2} Y_{s} \\
& \frac{d Y_{i}}{d t}=\beta_{2} Y_{s} X_{i}-\mu_{2} Y_{i}-\alpha_{2} Y_{i}, \\
& \frac{d Y_{r}}{d t}=k \beta_{2} Y_{s} X_{r}-\mu_{2} Y_{r}-k_{2} \alpha_{2} Y_{r} .
\end{aligned}
$$

Using standard methods, it is easy to see that disease free equilibrium $E_{0}=\left(\Lambda_{1} / \mu_{1}, 0,0, \Lambda_{2} / \mu_{2}, 0,0\right)$ always exists. Let $X_{s 0}=\Lambda_{1} / \mu_{1}, Y_{s 0}=\Lambda_{2} / \mu_{2}, m_{1}=\mu_{1}+\alpha_{1}+\sigma, m_{2}=\mu_{1}+k_{1} \alpha_{1}$, $m_{3}=\mu_{2}+\alpha_{2}$, and $m_{4}=\mu_{2}+k_{2} \alpha_{2}$. According to the concept of next generation matrix [21] and the formula of the basic 
reproductive number for ODE compartmental models [22], if we let

$$
\begin{gathered}
F=\left(\begin{array}{cccc}
0 & 0 & \beta_{1} X_{s 0} & 0 \\
0 & 0 & 0 & k \beta_{1} X_{s 0} \\
\beta_{2} Y_{s 0} & 0 & 0 & 0 \\
0 & k \beta_{2} Y_{s 0} & 0 & 0
\end{array}\right), \\
V=\left(\begin{array}{cccc}
m_{1} & 0 & 0 & 0 \\
0 & m_{2} & 0 & 0 \\
0 & 0 & m_{3} & 0 \\
0 & 0 & 0 & m_{4}
\end{array}\right)
\end{gathered}
$$

one can calculate that the eigenvalues of the next generation matrix $F V^{-1}$ are given by

$$
R_{0 i}=\sqrt{\frac{\beta_{1} \beta_{2} X_{s 0} Y_{s 0}}{m_{1} m_{3}}}, \quad R_{0 r}=\sqrt{\frac{k^{2} \beta_{1} \beta_{2} X_{s 0} Y_{s 0}}{m_{2} m_{4}}} .
$$

Then, it follows that the basic reproductive number for the system (1) is given by

$$
R_{0}=\max \left\{R_{0 i}, R_{0 r}\right\} .
$$

To obtain other equilibria, we let the right-hand side of (1) equal to zero and obtain

$$
\begin{gathered}
\Lambda_{1}-\beta_{1} X_{s} Y_{i}-k \beta_{1} X_{s} Y_{r}-\mu_{1} X_{s}+(1-\rho) \sigma X_{i}=0, \\
\beta_{1} X_{s} Y_{i}-\mu_{1} X_{i}-\alpha_{1} X_{i}-\sigma X_{i}=0, \\
k \beta_{1} X_{s} Y_{r}+\rho \sigma X_{i}-\mu_{1} X_{r}-k_{1} \alpha_{1} X_{r}=0, \\
\Lambda_{2}-\beta_{2} Y_{s} X_{i}-k \beta_{2} Y_{s} X_{r}-\mu_{2} Y_{s}=0, \\
\beta_{2} Y_{s} X_{i}-\mu_{2} Y_{i}-\alpha_{2} Y_{i}=0, \\
k \beta_{2} Y_{s} X_{r}-\mu_{2} Y_{r}-k_{2} \alpha_{2} Y_{r}=0 .
\end{gathered}
$$

If $X_{i}=0$, we have $Y_{i}=0$ and a formula of $X_{r}$ as follows:

$$
\begin{array}{r}
\left(k^{2} \beta_{1} \beta_{2} \Lambda_{2} m_{2}+\mu_{1} m_{2} m_{4} k \beta_{2}\right) X_{r} \\
=k^{2} \beta_{1} \beta_{2} \Lambda_{1} \Lambda_{2}-\mu_{1} \mu_{2} m_{2} m_{4} .
\end{array}
$$

Note that $R_{0 r}>1$ equals to $k^{2} \beta_{1} \beta_{2} \Lambda_{1} \Lambda_{2}>\mu_{1} \mu_{2} m_{2} m_{4}$. Hence, if $R_{0 r}>1$, there exists a boundary equilibrium with only resistant type, given as $E_{0 r}=\left(X_{s r}, 0, X_{r r}, Y_{s r}, 0, Y_{r r}\right)$, where

$$
\begin{aligned}
& X_{s r}=\frac{\Lambda_{1}\left(\mu_{1} m_{4} R_{0 r}^{2}+k \beta_{1} \Lambda_{2}\right)}{\left(\mu_{1}^{2} m_{4}+k \beta_{1} \mu_{1} \Lambda_{2}\right) R_{0 r}^{2}}, \\
& X_{r r}=\frac{\mu_{1} \mu_{2} m_{4}\left(R_{0 r}^{2}-1\right)}{\left(\mu_{1} m_{4}+k \beta_{1} \Lambda_{2}\right) k \beta_{2}}, \\
& Y_{s r}=\frac{\Lambda_{2}\left(\mu_{1} m_{4}+k \beta_{1} \Lambda_{2}\right)}{\mu_{1} \mu_{2} m_{4} R_{0 r}^{2}+k \beta_{1} \Lambda_{2} \mu_{2}}, \\
& Y_{r r}=\frac{\Lambda_{2} \mu_{1}\left(R_{0 r}^{2}-1\right)}{\mu_{1} m_{4} R_{0 r}^{2}+k \beta_{1} \Lambda_{2}} .
\end{aligned}
$$

If drug treatment does not lead to drug resistance, that is, $\rho=0$, then when $R_{0 i}>1$, we can obtain the other boundary equilibrium with only sensitive type, given as $E_{0 i}=$ $\left(X_{s i}, X_{i i}, 0, Y_{s i}, Y_{i i}, 0\right)$, where

$$
\begin{aligned}
& X_{s i}=\frac{\Lambda_{1} m_{1} m_{3}+\left(\mu_{1}+\alpha_{1}\right) \mu_{2} m_{1} m_{3}}{\beta_{1} \beta_{2} \Lambda_{2}\left(\mu_{1}+\alpha_{1}\right)+\mu_{1} m_{1} m_{3}}, \\
& X_{i i}=\frac{\mu_{1} \mu_{2} m_{1} m_{3}\left(R_{0 i}^{2}-1\right)}{\beta_{1} \beta_{2} \Lambda_{2}\left(\mu_{1}+\alpha_{1}\right)+\mu_{1} m_{1} m_{3}}, \\
& Y_{s i} \\
& =\frac{\beta_{1} \beta_{2} \Lambda_{2}^{2}\left(\mu_{1}+\alpha_{1}\right)+\Lambda_{2} \mu_{1} m_{1} m_{3}}{\beta_{2} \mu_{1} \mu_{2} m_{1} m_{3}\left(R_{0 i}^{2}-1\right)+\beta_{1} \beta_{2} \Lambda_{2} \mu_{2}\left(\mu_{1}+\alpha_{1}\right)+\mu_{1} \mu_{2} m_{1} m_{3}}, \\
& Y_{i i} \\
& =\frac{\Lambda_{2} \beta_{2} \mu_{1} \mu_{2} m_{1}\left(R_{0 i}^{2}-1\right)}{\beta_{2} \mu_{1} \mu_{2} m_{1} m_{3}\left(R_{0 i}^{2}-1\right)+\beta_{1} \beta_{2} \Lambda_{2} \mu_{2}\left(\mu_{1}+\alpha_{1}\right)+\mu_{1} \mu_{2} m_{1} m_{3}} .
\end{aligned}
$$

Now, we study existence of coexistence equilibrium for the system (1). From (5)-(10), we obtain

$$
\begin{aligned}
{\left[\beta_{1} \beta_{2} \Lambda_{2}\right.} & \left.\left(\mu_{1}+\alpha_{1}\right)+\mu_{1} m_{1} m_{3} \beta_{2}\right] X_{i} \\
& +\left(\mu_{1} m_{1} m_{3} k \beta_{2}+\beta_{1} \beta_{2} \Lambda_{2} m_{2}\right) X_{r} \\
= & \beta_{1} \beta_{2} \Lambda_{1} \Lambda_{2}-\mu_{1} \mu_{2} m_{1} m_{3} .
\end{aligned}
$$

Following (6), (7), (9), and (10), we have

$$
\frac{Y_{i}}{Y_{r}}=\frac{k m_{1} X_{i}}{m_{2} X_{r}-\rho \sigma X_{i}}=\frac{m_{4} X_{i}}{k m_{3} X_{r}}
$$

In the case that $\rho=0$, (15) leads to

$$
\frac{k m_{1} X_{i}}{m_{2} X_{r}}=\frac{m_{4} X_{i}}{k m_{3} X_{r}}
$$

Note that $R_{0 i}=R_{0 r}$ equals to $\mathrm{km} / \mathrm{m}_{2}=\mathrm{m}_{4} / \mathrm{km}_{3}$. It is easy to see that if $R_{0 i} \neq R_{0 r}$, (16) cannot hold, which implies that there is not coexistence equilibrium. If $R_{0 i}=R_{0 r},(16)$ always holds. Hence, if $R_{0 r}=R_{0 i}>1$, that is, $\beta_{1} \beta_{2} \Lambda_{1} \Lambda_{2}>\mu_{1} \mu_{2} m_{1} m_{3}$, (14) represents the existence of coexistence equilibrium in the form of a line.

In the case that $\rho \neq 0$, (15) leads to

$$
m_{4} \rho \sigma X_{i}=\left(m_{2} m_{4}-k^{2} m_{1} m_{3}\right) X_{r} .
$$

Note that $R_{0 i}>R_{0 r}$ equals to $m_{2} m_{4}-k^{2} m_{1} m_{3}>0$. Following (14) and (17), we can obtain that if $R_{0 i}>R_{0 r}$ and $R_{0 i}>1$, 
then the unique coexistence equilibrium is given by $E_{c}=$ $\left(X_{s c}, X_{i c}, X_{r c}, Y_{s c}, Y_{i c}, Y_{r c}\right)$, where

$$
\begin{aligned}
X_{r c}= & m_{4} \rho \sigma\left(\beta_{1} \beta_{2} \Lambda_{1} \Lambda_{2}-\mu_{1} \mu_{2} m_{1} m_{3}\right) \\
& \times\left(\left[\beta_{1} \beta_{2} \Lambda_{2}\left(\mu_{1}+\alpha_{1}\right)+\mu_{1} m_{1} m_{3} \beta_{2}\right]\right. \\
& \quad \times\left(m_{2} m_{4}-k^{2} m_{1} m_{3}\right) \\
& \left.\quad+m_{4} \rho \sigma\left(\mu_{1} m_{1} m_{3} k \beta_{2}+\beta_{1} \beta_{2} \Lambda_{2} m_{2}\right)\right)^{-1}, \\
X_{i c}= & \frac{m_{2} m_{4}-k^{2} m_{1} m_{3}}{m_{4} \rho \sigma} X_{r c}, \\
Y_{s c}= & \frac{\Lambda_{2}}{\beta_{2} X_{i c}+k \beta_{2} X_{r c}+\mu_{2}}, \\
Y_{r c}= & \frac{k \Lambda_{2} \beta_{2} X_{r c}}{m_{4}\left(\beta_{2} X_{i c}+k \beta_{2} X_{r c}+\mu_{2}\right)}, \\
Y_{i c}= & \frac{\beta_{2} Y_{s c} X_{i c}}{\mu_{2}+\alpha_{2}}, \\
X_{s c}= & \frac{\Lambda_{1}+(1-\rho) \sigma X_{i c}}{\beta_{1} Y_{i c}+k \beta_{1} Y_{r c}+\mu_{1}} .
\end{aligned}
$$

Summarizing above analyses, we have the following result.

$$
J=\left(\begin{array}{cccccc}
-\beta_{1} Y_{i}-k \beta_{1} Y_{r}-\mu_{1} & (1-\rho) \sigma & 0 & 0 & -\beta_{1} X_{s} & -k \beta_{1} X_{s} \\
\beta_{1} Y_{i} & -m_{1} & 0 & 0 & \beta_{1} X_{s} & 0 \\
k \beta_{1} Y_{r} & \rho \sigma & -m_{2} & 0 & 0 & k \beta_{1} X_{s} \\
0 & -\beta_{2} Y_{s} & -k \beta_{2} Y_{s} & -\beta_{2} X_{i}-k \beta_{2} X_{r}-\mu_{2} & 0 & 0 \\
0 & \beta_{2} Y_{s} & 0 & \beta_{2} X_{i} & -m_{3} & 0 \\
0 & 0 & k \beta_{2} Y_{s} & k \beta_{2} X_{r} & 0 & -m_{4}
\end{array}\right) .
$$

Then, the eigenvalues of $E_{0}$ are $-\mu_{1},-\mu_{2}$ and roots of the following equations:

$$
\begin{aligned}
\lambda^{2}+\left(m_{1}+m_{3}\right) \lambda+m_{1} m_{3}-\beta_{1} \beta_{2} X_{s 0} Y_{s 0} & =0, \\
\lambda^{2}+\left(m_{2}+m_{4}\right) \lambda+m_{2} m_{4}-k^{2} \beta_{1} \beta_{2} X_{s 0} Y_{s 0} & =0 .
\end{aligned}
$$

Note that $R_{0}<1$ equals to $R_{0 i}<1$ and $R_{0 r}<1$, which leads to $m_{1} m_{3}-\beta_{1} \beta_{2} X_{s 0} Y_{s 0}>0$ and $m_{2} m_{4}-k^{2} \beta_{1} \beta_{2} X_{s 0} Y_{s 0}>0$. Hence, if $R_{0}<1$, then all the roots of (20) have negative real parts. Hence, using the Routh-Hurwitz criterion, we can obtain that the disease free equilibrium $E_{0}$ of the system (1) is locally asymptotically stable if $R_{0}<1$ and unstable if $R_{0}>1$.

Now, we turn to the study of the global stability of the disease free equilibrium of the model (1) by using Metzler matrix theory and the technique of Kamgang and Sallet [23].
Lemma 1. The existence of equilibria for the system (1) is as follows:

(1) the disease free equilibrium $E_{0}$ always exists;

(2) if $R_{0 r}>1$, there exists a boundary equilibrium with only resistant type $E_{0 r}$;

(3) if $\rho=0$ and $R_{0 i}>1$, there exists a boundary equilibrium with only sensitive type $E_{0 i}$;

(4) if $\rho=0$ and $R_{0 i}=R_{0 r}>1$, there exists coexistence equilibrium in the form of a line (14);

(5) if $\rho \neq 0$ and $R_{0 i}>R_{0 r}$ and $R_{0 i}>1$, there exists a unique coexistence equilibrium $E_{c}$.

The following section shows that the basic reproductive number $R_{0}$ provides a threshold condition for schistosoma extinction in (1).

\section{Stability Analysis of the Disease Free Equilibrium}

In this section, we will analyze stability of the disease free equilibrium of the model (1). The stability of the disease free equilibrium determines whether schistosomiasis will be permanent in an uninfected population. The following result shows that schistosome will go extinct if $R_{0}<1$.

Theorem 2. The disease free equilibrium $E_{0}$ of the system (1) is locally asymptotically stable if $R_{0}<1$ and unstable if $R_{0}>1$.

Proof. The Jacobian matrix for the system (1) is given by

Consider systems of the following form:

$$
\begin{aligned}
& \frac{d x_{1}}{d t}=f\left(x_{1}, x_{2}\right), \\
& \frac{d x_{2}}{d t}=g\left(x_{1}, x_{2}\right),
\end{aligned}
$$

where $x_{1} \in R_{+}^{n_{1}}, x_{2} \in R_{+}^{n_{2}}$, and $f$ and $g$ are $C^{1}$. We denote by $x=\left(x_{1}, x_{2}\right)$ the state of the system and $\left(x_{1}^{*}, 0\right)$ is a disease free equilibrium on a positively invariant set $\Omega \subset R_{+}^{n_{1}+n_{2}}$. Now rewrite (21) as

$$
\begin{aligned}
& \frac{d x_{1}}{d t}=A_{1}(x) \cdot\left(x_{1}-x_{1}^{*}\right)+A_{12}(x) \cdot x_{2}, \\
& \frac{d x_{2}}{d t}=A_{2}(x) x_{2} .
\end{aligned}
$$


For the system (22), we make the following assumptions.

$\left(h_{1}\right)$ The system is defined on the positively invariant set $\Omega$ of the nonnegative orthant. The system is dissipative on $\Omega$.

$\left(\mathrm{h}_{2}\right)$ The subsystem $d x_{1} / d t=A_{1}(x) \cdot\left(x_{1}-x_{1}^{*}\right)$ is globally asymptotically stable at the equilibrium $x_{1}^{*}$ on the canonical projection of $\Omega$ on $R_{+}^{n_{1}}$.

$\left(\mathrm{h}_{3}\right)$ The matrix $A_{2}(x)$ is Metzler and irreducible for any given $x \in \Omega$.

$\left(\mathrm{h}_{4}\right)$ There exists an maximum matrix $\bar{A}_{2}$, then for any $\bar{x} \epsilon$ $\Omega$ such that $\bar{A}_{2}=A_{2}(\bar{x}), \bar{x} \in R_{+}^{n_{1}} \times\{0\}$.

$\left(\mathrm{h}_{5}\right) \alpha\left(\bar{A}_{2}\right) \leq 0$, that is, the greatest real part of eigenvalues of $\bar{A}_{2}$ is nonnegative.

For convenience, we state two lemmas due to Kamgang and Sallet [23].

Lemma 3. If the above hypotheses, $h_{1}-h_{5}$, are satisfied, then the disease free equilibrium is globally asymptotically stable in $\Omega$.

Lemma 4. If the same notations and hypotheses in Lemma 3 hold and if, furthermore, we have $\bar{A}_{2}=A_{2}\left(x_{1}^{*}, 0\right)$, the disease free equilibrium is globally asymptotically stable if and only if $\alpha\left(\bar{A}_{2}\right) \leq 0$.

Next, we discuss the global stability of the disease free equilibrium $E_{0}$ of the system (1) using the above two Lemmas.

From the system (1), we know

$$
\begin{aligned}
& \frac{d\left(X_{s}+X_{i}+X_{r}\right)}{d t} \\
& \quad=\Lambda_{1}-\mu_{1}\left(X_{s}+X_{i}+X_{r}\right)-\alpha_{1} X_{i}-k_{2} \alpha_{1} X_{r} \\
& \quad \leq \Lambda_{1}-\mu_{1}\left(X_{s}+X_{i}+X_{r}\right), \\
& \frac{d\left(Y_{s}+Y_{i}+Y_{r}\right)}{d t} \\
& \quad=\Lambda_{2}-\mu_{2}\left(Y_{s}+Y_{i}+Y_{r}\right)-\alpha_{2} Y_{i}-k_{4} \alpha_{2} Y_{r} \\
& \leq \Lambda_{2}-\mu_{2}\left(Y_{s}+Y_{i}+Y_{r}\right) .
\end{aligned}
$$

This proves that the set

$$
\begin{gathered}
\Omega=\left\{\left(X_{s}, X_{i}, X_{r}, Y_{s}, Y_{i}, Y_{r}\right) \in \mathbb{R}_{+}^{5} \mid X_{s}+X_{i}+X_{r}\right. \\
\left.\leq \frac{\Lambda_{1}}{\mu_{1}}, Y_{s}+Y_{i}+Y_{r} \leq \frac{\Lambda_{2}}{\mu_{2}}\right\}
\end{gathered}
$$

is a compact positively invariant absorbing set contained in the nonnegative orthant. Thus, the system (1) is dissipative on $\Omega$ because the trajectories of (1) are forward bounded. Now, we will study the system (1) on $\Omega$.
We set for system (1) $x_{1}=\left(X_{s}, Y_{s}\right), x_{2}=\left(X_{i}, X_{r}, Y_{i}, Y_{r}\right)$, and $x_{1}^{*}=\left(\Lambda_{1} / \mu_{1}, \Lambda_{2} / \mu_{2}\right)$. As in [23], we express the subsystem as $d x_{1} / d t=A_{1}\left(x_{1}, 0\right)\left(x_{1}-x_{1}^{*}\right)$ and

$$
\begin{aligned}
& \frac{d X_{s}}{d t}=\Lambda_{1}-\mu_{1} X_{s}, \\
& \frac{d Y_{s}}{d t}=\Lambda_{2}-\mu_{2} Y_{s} .
\end{aligned}
$$

This is a linear system, and its unique equilibrium $\left(A_{1} / \mu_{1}\right.$, $A_{2} / \mu_{2}$ ) (corresponding to the disease free equilibrium of (1)) is globally asymptotically stable, hence the assumptions $\left(\mathrm{h}_{1}\right)$ and $\left(\mathrm{h}_{2}\right)$ are satisfied.

The matrix $A_{2}(x)$ is given by

$$
A_{2}(x)=\left(\begin{array}{cccc}
-m_{1} & 0 & \beta_{1} X_{s} & 0 \\
\rho \sigma & -m_{2} & 0 & k \beta_{1} X_{s} \\
\beta_{2} Y_{s} & 0 & -m_{3} & 0 \\
0 & k \beta_{2} Y_{s} & 0 & -m_{4}
\end{array}\right) .
$$

As required by hypothesis $\mathrm{h}_{3}$, for any $x \in \Omega$, the matrix $A_{2}(x)$ is irreducible.

Now, let us check $\left(\mathrm{h}_{4}\right)$. There is a maximum which is uniquely realized in $\Omega$ if $X_{s}=\Lambda_{1} / \mu_{1}$ and $Y_{s}=\Lambda_{2} / \mu_{2}$, which corresponds to the disease free equilibrium. This maximum matrix is then $J_{2}$, the subblock of the Jacobian matrix at the disease free equilibrium, corresponding to the matrix $A_{2}(x)$. The matrix $J_{2}$ is given by

$$
J_{2}=\left(\begin{array}{cccc}
-m_{1} & 0 & \beta_{1} X_{s 0} & 0 \\
\rho \sigma & -m_{2} & 0 & k \beta_{1} X_{s 0} \\
\beta_{2} Y_{s 0} & 0 & -m_{3} & 0 \\
0 & k \beta_{2} Y_{s 0} & 0 & -m_{4}
\end{array}\right)
$$

Therefore, we are in the situation of Lemma 4 , where the maximum is attained at the disease free equilibrium.

The hypothesis $\left(\mathrm{h}_{5}\right)$ requires that $\alpha\left(J_{2}\right) \leq 0$. Writing $J_{2}$ as a block matrix $J_{2}=\left(\begin{array}{ll}A_{2 \times 2} & B_{2 \times 2} \\ C_{2 \times 2} & D_{2 \times 2}\end{array}\right)$. Since $A$ is already a Metzler stable matrix, the condition $\alpha\left(J_{2}\right) \leq 0$ is equivalent to the condition $\alpha\left(D-C A^{-1} B\right) \leq 0$ [23], where

$$
D-C A^{-1} B=\left(\begin{array}{cc}
\frac{\beta_{1} \beta_{2} X_{s 0} Y_{s 0}}{m_{1}}-m_{3} & 0 \\
\frac{\rho \sigma k \beta_{1} \beta_{2} X_{s 0} Y_{s 0}}{m_{1} m_{2}} & \frac{k^{2} \beta_{1} \beta_{2} X_{s 0} Y_{s 0}}{m_{2}}-m_{4}
\end{array}\right) .
$$

Then,

$$
\begin{aligned}
\alpha & \left(D-C A^{-1} B\right) \\
& =\max \left\{\frac{\beta_{1} \beta_{2} X_{s 0} Y_{s 0}-m_{1} m_{3}}{m_{1}}, \frac{k^{2} \beta_{1} \beta_{2} X_{s 0} Y_{s 0}-m_{2} m_{4}}{m_{2}}\right\} .
\end{aligned}
$$

Hence, the condition $\alpha\left(D-C A^{-1} B\right) \leq 0$ is equivalent to $R_{0} \leq$ 1. We have seen that the hypotheses $\left(h_{1}\right),\left(h_{2}\right),\left(h_{3}\right),\left(h_{4}\right)$, and $\left(\mathrm{h}_{5}\right)$ are satisfied. Then, by Lemma 4 , we have the following result.

Theorem 5. The disease free equilibrium $E_{0}$ of the system (1) is globally asymptotically stable if $R_{0} \leq 1$. 


\section{Stability Analysis of the Boundary Equilibria}

In this section, we turn to study stability of the two boundary equilibria. From Lemma 1, we know that if $R_{0 r}>1$, there exists a boundary equilibrium with only resistant type $E_{0 r}$. Through calculations, we can obtain the characteristic equation as following:

$$
\begin{aligned}
\left\{\left(\lambda+m_{1}\right)\left(\lambda+m_{3}\right)-\beta_{1} \beta_{2} X_{s r} Y_{s r}\right\} & \\
\times\{ & \left(\lambda+m_{2}\right)\left(\lambda+m_{4}\right)\left(\lambda+\mu_{1}+k \beta_{1} Y_{r r}\right) \\
& \times\left(\lambda+\mu_{2}+k \beta_{2} X_{r r}\right) \\
& \left.-k^{2} \beta_{1} \beta_{2} X_{s r} Y_{s r}\left(\lambda+\mu_{1}\right)\left(\lambda+\mu_{2}\right)\right\}=0 .
\end{aligned}
$$

Hence, the eigenvalue of $E_{0 r}$ are roots of the following equations:

$$
\begin{gathered}
\lambda^{2}+\left(m_{1}+m_{3}\right) \lambda+m_{1} m_{3}-\beta_{1} \beta_{2} X_{s r} Y_{s r}=0 \\
\lambda^{4}+a_{1} \lambda^{3}+a_{2} \lambda^{2}+a_{3} \lambda+a_{4}=0
\end{gathered}
$$

Here,

$$
\begin{aligned}
a_{1}= & m_{2}+m_{4}+\frac{\Lambda_{1}}{X_{s r}}+\frac{\Lambda_{2}}{Y_{s r}}>0, \\
a_{2}= & m_{2} m_{4}+\left(m_{2}+m_{4}\right)\left(\frac{\Lambda_{1}}{X_{s r}}+\frac{\Lambda_{2}}{Y_{s r}}\right) \\
& +\frac{\Lambda_{1}}{X_{s r}} \frac{\Lambda_{2}}{Y_{s r}}-k^{2} \beta_{1} \beta_{2} X_{s r} Y_{s r}, \\
a_{3}= & m_{2} m_{4}\left(\frac{\Lambda_{1}}{X_{s r}}+\frac{\Lambda_{2}}{Y_{s r}}\right)+\left(m_{2}+m_{4}\right) \frac{\Lambda_{1}}{X_{s r}} \frac{\Lambda_{2}}{Y_{s r}} \\
& -k^{2} \beta_{1} \beta_{2} X_{s r} Y_{s r}\left(\mu_{1}+\mu_{2}\right), \\
a_{4}= & m_{2} m_{4} \frac{\Lambda_{1}}{X_{s r}} \frac{\Lambda_{2}}{Y_{s r}}-k^{2} \beta_{1} \beta_{2} X_{s r} Y_{s r} \mu_{1} \mu_{2} .
\end{aligned}
$$

From (12), we can obtain $X_{s r} Y_{s r}=X_{s 0} Y_{s 0} / R_{0 r}^{2}=$ $m_{2} m_{4} / k^{2} \beta_{1} \beta_{2}$. Then,

$$
m_{1} m_{3}-\beta_{1} \beta_{2} X_{s r} Y_{s r}=m_{1} m_{3}-\frac{m_{2} m_{4}}{k^{2}}
$$

It is easy to see that $R_{0 i}^{2} / R_{0 r}^{2}=\left(m_{2} m_{4} / k^{2}\right) / m_{1} m_{3}$. Then, $R_{0 i}<R_{0 r}$ equals to $m_{1} m_{3}>\beta_{1} \beta_{2} X_{s r} Y_{s r}$, which implies that the roots of (31) have negative real parts if $R_{0 i}<R_{0 r}$.
Based on $\left(\Lambda_{1} / X_{s r}\right)\left(\Lambda_{2} / Y_{s r}\right)=\mu_{1} \mu_{2} R_{0 r}^{2}$ and $k^{2} \beta_{1} \beta_{2} X_{s r} Y_{s r}=m_{2} m_{4}$, we can obtain

$$
\begin{aligned}
a_{2}= & \left(m_{2}+m_{4}\right)\left(\frac{\Lambda_{1}}{X_{s r}}+\frac{\Lambda_{2}}{Y_{s r}}\right)+\frac{\Lambda_{1}}{X_{s r}} \frac{\Lambda_{2}}{Y_{s r}}>0, \\
a_{3}= & m_{2} m_{4}\left[\frac{k \beta_{1} \mu_{1} \Lambda_{2}\left(R_{0 r}^{2}-1\right)}{\mu_{1} m_{4} R_{0 r}^{2}+k \beta_{1} \Lambda_{2}}+\frac{\mu_{1} \mu_{2} m_{4}\left(R_{0 r}^{2}-1\right)}{\mu_{1} m_{4}+k \beta_{1} \Lambda_{2}}\right] \\
& +\left(m_{2}+m_{4}\right) \mu_{1} \mu_{2} R_{0 r}^{2}, \\
a_{4}= & m_{2} m_{4} \mu_{1} \mu_{2}\left(R_{0 r}^{2}-1\right) .
\end{aligned}
$$

Here, $a_{3}>0$ and $a_{4}>0$ if $R_{0 r}>1$.

If $R_{0 r}>1$, let $k \beta_{1} \mu_{1} \Lambda_{2}\left(R_{0 r}^{2}-1\right) /\left(\mu_{1} m_{4} R_{0 r}^{2}+k \beta_{1} \Lambda_{2}\right)=$ $p_{1}>0$ and $\mu_{1} \mu_{2} m_{4}\left(R_{0 r}^{2}-1\right) /\left(\mu_{1} m_{4}+k \beta_{1} \Lambda_{2}\right)=p_{2}>0$, then $\Lambda_{1} / X_{s r}=p_{1}+\mu_{1}>\mu_{1}$ and $\Lambda_{2} / Y_{s r}=p_{2}+\mu_{2}>\mu_{2}$. Note that $m_{2}=\mu_{1}+k_{1} \alpha_{1}>\mu_{1}$ and $m_{4}=\mu_{2}+k_{2} \alpha_{2}>\mu_{2}$, then $m_{2}+m_{4}>\mu_{1}+\mu_{2}$ and $m_{2} m_{4}>\mu_{1} \mu_{2}$. Hence, we can obtain

$$
\begin{aligned}
& H_{1}=a_{1}>0 \text {, } \\
& H_{2}=\left|\begin{array}{cc}
a_{1} & a_{3} \\
1 & a_{2}
\end{array}\right|=a_{1} a_{2}-a_{3} \\
& =\left(m_{2}^{2}+m_{4}^{2}+m_{2} m_{4}+\mu_{1} \mu_{2} R_{0 r}^{2}\right)\left(\frac{\Lambda_{1}}{X_{s r}}+\frac{\Lambda_{2}}{Y_{s r}}\right) \\
& +\left(m_{2}+m_{4}\right)\left(\frac{\Lambda_{1}}{X_{s r}}+\frac{\Lambda_{2}}{Y_{s r}}\right)^{2}+m_{2} m_{4}\left(\mu_{1}+\mu_{2}\right)>0 \\
& H_{3}=\left|\begin{array}{ccc}
a_{1} & a_{3} & 0 \\
1 & a_{2} & 0 \\
0 & a_{1} & a_{3}
\end{array}\right|=a_{1} a_{2} a_{3}-a_{3}^{2}-a_{1}^{2} a_{4} \\
& =\left(m_{2}+m_{4}\right)^{2} m_{2} m_{4}\left(\frac{\Lambda_{1}}{X_{s r}}+\frac{\Lambda_{2}}{Y_{s r}}\right)^{2} \\
& +\left(m_{2}+m_{4}\right)^{3}\left(\frac{\Lambda_{1}}{X_{s r}}+\frac{\Lambda_{2}}{Y_{s r}}\right) \mu_{1} \mu_{2} R_{0 r}^{2} \\
& +\mu_{1} \mu_{2} R_{0 r}^{2}\left(m_{2}+m_{4}\right) m_{2} m_{4}\left(\frac{\Lambda_{1}}{X_{s r}}+\frac{\Lambda_{2}}{Y_{s r}}\right) \\
& +\left(m_{2}+m_{4}\right) m_{2} m_{4}\left(\frac{\Lambda_{1}}{X_{s r}}+\frac{\Lambda_{2}}{Y_{s r}}\right)^{3} \\
& +\left(m_{2}+m_{4}\right)^{2}\left(\frac{\Lambda_{1}}{X_{s r}}+\frac{\Lambda_{2}}{Y_{s r}}\right)^{2} \mu_{1} \mu_{2} R_{0 r}^{2} \\
& +\left(m_{2}+m_{4}\right)\left(\frac{\Lambda_{1}}{X_{s r}}+\frac{\Lambda_{2}}{Y_{s r}}\right) \mu_{1}^{2} \mu_{2}^{2} R_{0 r}^{4} \\
& +2\left(\mu_{1}+\mu_{2}\right) m_{2}^{2} m_{4}^{2}\left(\frac{\Lambda_{1}}{X_{s r}}+\frac{\Lambda_{2}}{Y_{s r}}\right) \\
& +\left(m_{2}+m_{4}\right) \mu_{1} \mu_{2} R_{0 r}^{2} m_{2} m_{4}\left(\mu_{1}+\mu_{2}\right) \\
& +\left(m_{2}+m_{4}\right)^{2} \mu_{1} \mu_{2} m_{2} m_{4}
\end{aligned}
$$




$$
\begin{aligned}
& +2\left(m_{2}+m_{4}\right)\left(\frac{\Lambda_{1}}{X_{s r}}+\frac{\Lambda_{2}}{Y_{s r}}\right) \mu_{1} \mu_{2} m_{2} m_{4} \\
& +\left(\frac{\Lambda_{1}}{X_{s r}}+\frac{\Lambda_{2}}{Y_{s r}}\right)^{2} \mu_{1} \mu_{2} m_{2} m_{4} \\
& -\left(m_{2}+m_{4}\right)^{2} m_{2} m_{4}\left(\frac{\Lambda_{1}}{X_{s r}}+\frac{\Lambda_{2}}{Y_{s r}}\right)\left(\mu_{1}+\mu_{2}\right) \\
& -\left(m_{2}+m_{4}\right) m_{2} m_{4}\left(\frac{\Lambda_{1}}{X_{s r}}+\frac{\Lambda_{2}}{Y_{s r}}\right)^{2}\left(\mu_{1}+\mu_{2}\right) \\
& -\mu_{1} \mu_{2} R_{0 r}^{2}\left(\mu_{1}+\mu_{2}\right) m_{2} m_{4}\left(\frac{\Lambda_{1}}{X_{s r}}+\frac{\Lambda_{2}}{Y_{s r}}\right) \\
& -\left(\frac{\Lambda_{1}}{X_{s r}}+\frac{\Lambda_{2}}{Y_{s r}}\right)^{2} m_{2}^{2} m_{4}^{2}-m_{2}^{2} m_{4}^{2}\left(\mu_{1}+\mu_{2}\right)^{2} \\
& -2 m_{2} m_{4}\left(m_{2}+m_{4}\right)\left(\frac{\Lambda_{1}}{X_{s r}}+\frac{\Lambda_{2}}{Y_{s r}}\right) \mu_{1} \mu_{2} R_{0 r}^{2} \\
& -m_{2} m_{4}\left(m_{2}+m_{4}\right)^{2} \mu_{1} \mu_{2} R_{0 r}^{2} \\
& >\left(m_{2}^{2}+m_{4}^{2}\right)^{2} m_{2} m_{4}\left(p_{1}^{2}+p_{2}^{2}+p_{1} p_{2}+p_{1} \mu_{1}+p_{2} \mu_{2}\right) \\
& +m_{2}^{2} m_{4}^{2}\left(p_{1}^{2}+p_{2}^{2}+2 p_{1} \mu_{1}+2 p_{2} \mu_{2}\right)>0, \\
& H_{4}=\left|\begin{array}{cccc}
a_{1} & a_{3} & 0 & 0 \\
1 & a_{2} & a_{4} & 0 \\
0 & a_{1} & a_{3} & 0 \\
0 & 1 & a_{2} & a_{4}
\end{array}\right|=a_{4} H_{3}>0 .
\end{aligned}
$$

It follows from Routh-Hurwitz criterion that all roots of (32) have negative real parts if $R_{0 r}>1$. Summering above analyses, we have the following result.

Theorem 6. The boundary equilibrium $E_{0 r}$ of the system (1) is locally asymptotically stable if $R_{0 r}>1$ and $R_{0 i}<R_{0 r}$.

Now, we study the global stability of the boundary equilibrium $E_{0 r}$. Consider the Lyapunov function

$$
V_{1}=d_{1} X_{i}+d_{2} Y_{i}
$$

where $d_{1}=\left(m_{3}+\beta_{2}\left(\Lambda_{2} / \mu_{2}\right)\right) / m_{1} m_{3}\left(R_{0 i}+1\right)$ and $d_{2}=\left(m_{1}+\right.$ $\left.\beta_{1}\left(\Lambda_{1} / \mu_{1}\right)\right) / m_{1} m_{3}\left(R_{0 i}+1\right)$. The Lyapunov derivative is

$$
\begin{aligned}
\frac{d V_{1}}{d t} & =d_{1}\left(\beta_{1} X_{s} Y_{i}-m_{1} X_{i}\right)+d_{2}\left(\beta_{2} Y_{s} X_{i}-m_{3} Y_{i}\right) \\
& \leq\left(d_{1} \beta_{1} \frac{\Lambda_{1}}{\mu_{1}}-d_{2} m_{3}\right) Y_{i}+\left(d_{2} \beta_{2} \frac{\Lambda_{2}}{\mu_{2}}-d_{1} m_{1}\right) X_{i} \\
& \leq\left(R_{0 i}-1\right)\left(Y_{i}+X_{i}\right) \\
& \leq 0 \text { for } R_{0 i} \leq 1 .
\end{aligned}
$$

This implies that the sensitive type dies out if $R_{0 i} \leq 1$. Then the largest compact invariant set of the system (1) in the set $\left\{d V_{1} / d t=0\right\}$ is $\Omega_{1}=\left(X_{s}, X_{i}, X_{r}, Y_{s}, Y_{i}, Y_{r}\right) \in \Omega \mid X_{i}=$ $Y_{i}=0$. Using the LaSalle-Lyapunov theorem, we know that all trajectories in $\Omega$ eventually tend to $\Omega_{1}$ as $t \rightarrow \infty$. Then, we only need to study the dynamical behavior of (1) in $\Omega_{1}$. At this time, (1) reduces to the following system

$$
\begin{aligned}
\frac{d X_{s}}{d t} & =\Lambda_{1}-k \beta_{1} X_{s} Y_{r}-\mu_{1} X_{s}, \\
\frac{d X_{r}}{d t} & =k \beta_{1} X_{s} Y_{r}-m_{2} X_{r}, \\
\frac{d Y_{s}}{d t} & =\Lambda_{2}-k \beta_{2} Y_{s} X_{r}-\mu_{2} Y_{s}, \\
\frac{d Y_{r}}{d t} & =k \beta_{2} Y_{s} X_{r}-m_{4} Y_{r} .
\end{aligned}
$$

To show that all trajectories of (39) in the interior of $\Omega_{1}$ approach the point $\left(X_{s r}, X_{r r}, Y_{s r}, Y_{r r}\right)$ corresponding to the boundary equilibrium $E_{0 r}$, consider the Lyapunov function

$$
\begin{aligned}
V_{2}=\bar{d}_{1}\{[ & \left.X_{s}-X_{s r}-X_{s r} \ln \left(\frac{X_{s}}{X_{s r}}\right)\right] \\
+ & {\left.\left[X_{r}-X_{r r}-X_{r r} \ln \left(\frac{X_{r}}{X_{r r}}\right)\right]\right\} } \\
+ & \bar{d}_{2}\left\{\left[Y_{s}-Y_{s r}-Y_{s r} \ln \left(\frac{Y_{s}}{Y_{s r}}\right)\right]\right. \\
+ & {\left.\left[Y_{r}-Y_{r r}-Y_{r r} \ln \left(\frac{Y_{r}}{Y_{r r}}\right)\right]\right\}, }
\end{aligned}
$$

where positive constants $\bar{d}_{1}$ and $\bar{d}_{2}$ are defined in the following. It is easy to see that $V_{2} \geq 0$ for $\left(X_{s}, X_{r}, Y_{s}, Y_{r}\right) \in \Omega_{1}$, and $V_{2}=0 \Leftrightarrow\left(X_{s}, X_{r}, Y_{s}, Y_{r}\right)=\left(X_{s r}, X_{r r}, Y_{s r}, Y_{r r}\right)$. Hence, the function $V_{2}$ is positive definite with respect to the point $\left(X_{s r}, X_{r r}, Y_{s r}, Y_{r r}\right)$.

Computing the derivative of $V_{2}$ along solutions of system (39), we have

$$
\begin{aligned}
\frac{d V_{2}}{d t}= & \bar{d}_{1}\left\{\left[\frac{d X_{s}}{d t}-\frac{d X_{s}}{d t}\left(\frac{X_{s r}}{X_{s}}\right)\right]+\left[\frac{d X_{r}}{d t}-\frac{d X_{r}}{d t}\left(\frac{X_{r r}}{X_{r}}\right)\right]\right\} \\
+ & \bar{d}_{2}\left\{\left[\frac{d Y_{s}}{d t}-\frac{d Y_{s}}{d t}\left(\frac{Y_{s r}}{Y_{s}}\right)\right]+\left[\frac{d Y_{r}}{d t}-\frac{d Y_{r}}{d t}\left(\frac{Y_{r r}}{Y_{r}}\right)\right]\right\} \\
= & \bar{d}_{1}\left[\Lambda_{1}-\mu_{1} X_{s}-\frac{X_{s r}}{X_{s}} \Lambda_{1}+k \beta_{1} X_{s r} Y_{r}+\mu_{1} X_{s r}\right. \\
& \left.-m_{2} X_{r}-k \beta_{1} X_{s} Y_{r} \frac{X_{r r}}{X_{r}}+m_{2} X_{r r}\right] \\
+ & \bar{d}_{2}\left[\Lambda_{2}-\mu_{2} Y_{s}-\frac{Y_{s r}}{Y_{s}} \Lambda_{2}+k \beta_{2} Y_{s r} X_{r}+\mu_{2} Y_{s r}\right. \\
& \left.-m_{4} Y_{r}-k \beta_{2} Y_{s} X_{r} \frac{Y_{r r}}{Y_{r}}+m_{4} Y_{r r}\right]
\end{aligned}
$$


Substituting $\Lambda_{1}=k \beta_{1} X_{s r} Y_{r r}+\mu_{1} X_{s r}, \Lambda_{2}=k \beta_{2} Y_{s r} X_{r r}+\mu_{2} Y_{s r}$, $m_{2} X_{r r}=k \beta_{1} X_{s r} Y_{r r}$ and $m_{4} Y_{r r}=k \beta_{2} Y_{s r} X_{r r}$ into $d V_{2} / d t$, we obtain

$$
\begin{aligned}
& \frac{d V_{2}}{d t} \\
& =\bar{d}_{1}\left\{\mu_{1} X_{s r}\left(2-\frac{X_{s}}{X_{s r}}-\frac{X_{s r}}{X_{s}}\right)+\left(k \beta_{1} X_{s r} Y_{r}-m_{2} X_{r}\right)\right. \\
& \left.+\left[2 k \beta_{1} X_{s r} Y_{r r}-k \beta_{1} Y_{r r} \frac{\left(X_{s r}\right)^{2}}{X_{s}}-k \beta_{1} X_{s} Y_{r} \frac{X_{r r}}{X_{r}}\right]\right\} \\
& +\bar{d}_{2}\left\{\mu_{2} Y_{s r}\left(2-\frac{Y_{s}}{Y_{s r}}-\frac{Y_{s r}}{Y_{s}}\right)+\left(k \beta_{2} Y_{s r} X_{r}-m_{4} Y_{r}\right)\right. \\
& \left.+\left[2 k \beta_{2} Y_{s r} X_{r r}-k \beta_{2} X_{r r} \frac{\left(Y_{s r}\right)^{2}}{Y_{s}}-k \beta_{2} Y_{s} X_{r} \frac{Y_{r r}}{Y_{r}}\right]\right\} \text {. }
\end{aligned}
$$

Let $\bar{d}_{1}=k \beta_{2} Y_{s r} X_{r r}$ and $\bar{d}_{2}=k \beta_{1} X_{s r} Y_{r r}$. Following from $m_{2} X_{r r}=k \beta_{1} X_{s r} Y_{r r}$ and $m_{4} Y_{r r}=k \beta_{2} Y_{s r} X_{r r}$, we have

$$
\bar{d}_{1}\left(k \beta_{1} X_{s r} Y_{r}-m_{2} X_{r}\right)+\bar{d}_{2}\left(k \beta_{2} Y_{s r} X_{r}-m_{4} Y_{r}\right)=0
$$

Hence, for all $\left(X_{s}, X_{r}, Y_{s}, Y_{r}\right) \in \Omega_{1}$,

$$
\begin{aligned}
\frac{d V_{2}}{d t}= & \bar{d}_{1}\left\{\mu_{1} X_{s r}\left(2-\frac{X_{s}}{X_{s r}}-\frac{X_{s r}}{X_{s}}\right)\right. \\
& \left.+k \beta_{1} X_{s r} Y_{r r}\left[2-\frac{X_{s r}}{X_{s}}-\frac{X_{s} Y_{r} X_{r r}}{X_{s r} Y_{r r} X_{r}}\right]\right\} \\
+ & \bar{d}_{2}\left\{\mu_{2} Y_{s r}\left(2-\frac{Y_{s}}{Y_{s r}}-\frac{Y_{s r}}{Y_{s}}\right)\right. \\
& \left.+k \beta_{2} Y_{s r} X_{r r}\left[2-\frac{Y_{s r}}{Y_{s}}-\frac{Y_{s} X_{r} Y_{r r}}{Y_{s r} X_{r r} Y_{r}}\right]\right\} \\
= & \bar{d}_{1} \mu_{1} X_{s r}\left(2-\frac{X_{s}}{X_{s r}}-\frac{X_{s r}}{X_{s}}\right)+\bar{d}_{2} \mu_{2} Y_{s r}\left(2-\frac{Y_{s}}{Y_{s r}}-\frac{Y_{s r}}{Y_{s}}\right) \\
+ & \bar{d}_{1} \bar{d}_{2}\left(4-\frac{X_{s r}}{X_{s}}-\frac{X_{s} Y_{r} X_{r r}}{X_{s r} Y_{r r} X_{r}}-\frac{Y_{s r}}{Y_{s}}-\frac{Y_{s} X_{r} Y_{r r}}{Y_{s r} X_{r r} Y_{r}}\right) \leq 0 .
\end{aligned}
$$

It is easy to see that

$$
\begin{gathered}
\frac{d V_{2}}{d t}=0 \Longleftrightarrow X_{s}=X_{s r}, \quad X_{r}=a X_{r r}, \\
Y_{s}=Y_{s r}, \quad Y_{r}=a Y_{r r},
\end{gathered}
$$

where $a$ is an arbitrary positive number. Substituting $X_{s}=$ $X_{s r}, X_{r}=a X_{r r}, Y_{s}=Y_{s r}$, and $Y_{r}=a Y_{r r}$ into the first equation of system (39), we obtain

$$
0=\Lambda_{1}-a k \beta_{1} X_{s r} Y_{r r}-\mu_{1} X_{s r}
$$

and then the above formula holds if and only if $a=1$. Therefore, the only compact invariant subset of the set where $d V_{2} / d t=0$ is the point $\left(X_{s r}, X_{r r}, Y_{s r}, Y_{r r}\right)$, corresponding to the boundary equilibrium $E_{0 r}$. By LaSalle's Invariance Principle, $E_{0 r}$ is globally asymptotically stable if $R_{0 r}>1$ and $R_{0 i} \leq 1$. Summering above analyses, we have the following result.

Theorem 7. The boundary equilibrium $E_{0 r}$ of the system (1) is globally asymptotically stable if $R_{0 r}>1 \geq R_{0 i}$.

Now, we turn to the other boundary equilibrium. From Lemma 1, we know that if $\rho=0$ and $R_{0 i}>1$, the boundary equilibrium with only sensitive type $E_{0 i}$ exists. Through calculations, we can obtain the characteristic equation as following:

$$
\begin{aligned}
& \left\{\left(\lambda+m_{2}\right)\left(\lambda+m_{4}\right)-k^{2} \beta_{1} \beta_{2} X_{s i} Y_{s i}\right\} \\
& \times\left\{\left(\lambda+m_{1}\right)\left(\lambda+m_{3}\right)\left(\lambda+\mu_{1}\right)\left(\lambda+\mu_{2}+\beta_{2} X_{i i}\right)\right. \\
& \quad+\beta_{1} Y_{i i}\left(\lambda+m_{3}\right)\left(\lambda+\mu_{2}+\beta_{2} X_{i i}\right)\left(\lambda+m_{1}-\sigma\right) \\
& \left.\quad-\beta_{1} \beta_{2} X_{s i} Y_{s i}\left(\lambda+\mu_{1}\right)\left(\lambda+\mu_{2}\right)\right\}=0 .
\end{aligned}
$$

Hence, the eigenvalue of $E_{0 i}$ are roots of the following equations:

$$
\begin{gathered}
\lambda^{2}+\left(m_{2}+m_{4}\right) \lambda+m_{2} m_{4}-k^{2} \beta_{1} \beta_{2} X_{s i} Y_{s i}=0 \\
\lambda^{4}+b_{1} \lambda^{3}+b_{2} \lambda^{2}+b_{3} \lambda+b_{4}=0 .
\end{gathered}
$$

Here,

$$
\begin{aligned}
b_{1}= & m_{1}+m_{3}+\mu_{1}+\mu_{2}+\beta_{2} X_{i i}+\beta_{1} Y_{i i}>0 \\
b_{2}= & \mu_{1}\left(m_{1}+m_{3}+\mu_{2}+\beta_{2} X_{i i}\right)+m_{1}\left(m_{3}+\mu_{2}+\beta_{2} X_{i i}\right) \\
& +m_{3}\left(\mu_{2}+\beta_{2} X_{i i}\right)+\beta_{1} Y_{i i}\left(m_{1}-\sigma+m_{3}+\mu_{2}+\beta_{2} X_{i i}\right) \\
& -m_{1} m_{3} \\
= & \mu_{1}\left(m_{1}+m_{3}+\mu_{2}+\beta_{2} X_{i i}\right)+m_{1}\left(\mu_{2}+\beta_{2} X_{i i}\right) \\
& +m_{3}\left(\mu_{2}+\beta_{2} X_{i i}\right)+\beta_{1} Y_{i i}\left(\mu_{1}+\alpha+m_{3}+\mu_{2}+\beta_{2} X_{i i}\right) \\
> & 0, \\
b_{3}= & \mu_{1} m_{1}\left(m_{3}+\mu_{2}+\beta_{2} X_{i i}\right)+m_{1} m_{3}\left(\mu_{2}+\beta_{2} X_{i i}\right) \\
& +\beta_{1} Y_{i i}\left[m_{3}\left(\mu_{2}+\beta_{2} X_{i i}\right)+\left(m_{1}-\sigma\right)\left(m_{3}+\mu_{2}+\beta_{2} X_{i i}\right)\right] \\
& -m_{1} m_{3}\left(\mu_{1}+\mu_{2}\right) \\
= & \mu_{1} m_{1}\left(\mu_{2}+\beta_{2} X_{i i}\right)+m_{1} m_{3} \beta_{2} X_{i i} \\
& +\beta_{1} Y_{i i}\left[m_{3}\left(\mu_{2}+\beta_{2} X_{i i}\right)+\left(\mu_{1}+\alpha\right)\left(m_{3}+\mu_{2}+\beta_{2} X_{i i}\right)\right] \\
> & 0, \\
b_{4}= & \mu_{1} m_{1} m_{3}\left(\mu_{2}+\beta_{2} X_{i i}\right)+\beta_{1} Y_{i i} m_{3}\left(\mu_{2}+\beta_{2} X_{i i}\right)\left(m_{1}-\sigma\right) \\
& -m_{1} m_{3} \mu_{1} \mu_{2} \\
= & \mu_{1} m_{1} m_{3} \beta_{2} X_{i i}+\beta_{1} Y_{i i} m_{3}\left(\mu_{2}+\beta_{2} X_{i i}\right)\left(\mu_{1}+\alpha\right)>0 . \\
&
\end{aligned}
$$


From (13), we can obtain that $\beta_{1} \beta_{2} X_{s i} Y_{s i}=m_{2} m_{4}$. Then, it is easy to see that the roots of (48) have negative real parts if $R_{0 i}>R_{0 r}$. Similarly to the case of $E_{0 r}$, using Routh-Hurtwitz criterion, we can obtain that all roots of (49) have negative real parts if $R_{0 i}>1$. Summering above analysis, we have the following result.

Theorem 8. When $\rho=0$, the boundary equilibrium $E_{0 i}$ of the system (1) is locally asymptotically stable if $R_{0 i}>1$ and $R_{0 i}>R_{0 r}$.

Now, we study the global stability of the boundary equilibrium $E_{0 i}$. Consider the Lyapunov function

$$
L_{1}=l_{1} X_{r}+l_{2} Y_{r} \text {, }
$$

where $l_{1}=\left(m_{4}+k \beta_{2}\left(\Lambda_{2} / \mu_{2}\right)\right) / m_{2} m_{4}\left(R_{0 r}+1\right)$ and $l_{2}=\left(m_{2}+\right.$ $\left.k \beta_{1}\left(\Lambda_{1} / \mu_{1}\right)\right) / m_{2} m_{4}\left(R_{0 r}+1\right)$. The Lyapunov derivative is

$$
\begin{aligned}
\frac{d L_{1}}{d t} & =l_{1}\left(k \beta_{1} X_{s} Y_{r}-m_{2} X_{r}\right)+l_{2}\left(k \beta_{2} Y_{s} X_{r}-m_{3} Y_{r}\right) \\
& \leq\left(l_{1} k \beta_{1} \frac{\Lambda_{1}}{\mu_{1}}-l_{2} m_{4}\right) Y_{r}+\left(l_{2} k \beta_{2} \frac{\Lambda_{2}}{\mu_{2}}-l_{1} m_{2}\right) X_{r} \\
& \leq\left(R_{0 r}-1\right)\left(Y_{r}+X_{r}\right) \\
& \leq 0 \text { for } R_{0 r} \leq 1 .
\end{aligned}
$$

This implies that the resistant type dies out if $R_{0 r} \leq 1$. Then, the largest compact invariant set of the system (1) in the set $\left\{d L_{1} / d t=0\right\}$ is $\Omega_{2}=\left\{\left(X_{s}, X_{i}, X_{r}, Y_{s}, Y_{i}, Y_{r}\right) \in \Omega \mid X_{r}=\right.$ $\left.Y_{r}=0\right\}$. Using the LaSalle-Lyapunov theorem, we know that all trajectories in $\Omega$ eventually tend to $\Omega_{2}$ as $t \rightarrow \infty$. Then, we only need to study the dynamical behavior of (1) in $\Omega_{2}$. At this time, (1) reduces to the following system:

$$
\begin{gathered}
\frac{d X_{s}}{d t}=\Lambda_{1}-\beta_{1} X_{s} Y_{i}-\mu_{1} X_{s}+\sigma X_{i} \\
\frac{d X_{i}}{d t}=\beta_{1} X_{s} Y_{i}-m_{1} X_{i} \\
\frac{d Y_{s}}{d t}=\Lambda_{2}-\beta_{2} Y_{s} X_{i}-\mu_{2} Y_{s} \\
\frac{d Y_{i}}{d t}=\beta_{2} Y_{s} X_{i}-m_{3} Y_{i} .
\end{gathered}
$$

To show that all trajectories of (53) in the interior of $\Omega_{2}$ approach the point $\left(X_{s i}, X_{i i}, Y_{s i}, Y_{i i}\right)$ corresponding to the boundary equilibrium $E_{0 i}$, consider the Lyapunov function

$$
\begin{aligned}
L_{2}=\bar{l}_{1}\left\{\left[X_{s}-X_{s i}-X_{s i} \ln \left(\frac{X_{s}}{X_{s i}}\right)\right]\right. \\
\left.+\left[X_{i}-X_{i i}-X_{i i} \ln \left(\frac{X_{i}}{X_{i i}}\right)\right]\right\} \\
+\bar{l}_{2}\left\{\left[Y_{s}-Y_{s i}-Y_{s i} \ln \left(\frac{Y_{s}}{Y_{s i}}\right)\right]\right. \\
\left.+\left[Y_{i}-Y_{i i}-Y_{i i} \ln \left(\frac{Y_{i}}{Y_{i i}}\right)\right]\right\},
\end{aligned}
$$

where positive constants $\bar{l}_{1}$ and $\bar{l}_{2}$ are defined in the following. It is easy to see that $L_{2} \geq 0$ for $\left(X_{s}, X_{i}, Y_{s}, Y_{i}\right) \in \Omega_{1}$ and $L_{2}=0 \Leftrightarrow\left(X_{s}, X_{i}, Y_{s}, Y_{i}\right)=\left(X_{s i}, X_{i i}, Y_{s i}, Y_{i i}\right)$. Hence, the function $L_{2}$ is positive definite with respect to the point $\left(X_{s i}, X_{i i}, Y_{s i}, Y_{i i}\right)$.

Computing the derivative of $L_{2}$ along solutions of system (39), we have

$$
\begin{aligned}
\frac{d L_{2}}{d t}=\bar{l}_{1}\left\{\left[\frac{d X_{s}}{d t}-\frac{d X_{s}}{d t}\left(\frac{X_{s i}}{X_{s}}\right)\right]+\left[\frac{d X_{i}}{d t}-\frac{d X_{i}}{d t}\left(\frac{X_{i i}}{X_{i}}\right)\right]\right\} \\
+\bar{l}_{2}\left\{\left[\frac{d Y_{s}}{d t}-\frac{d Y_{s}}{d t}\left(\frac{Y_{s i}}{Y_{s}}\right)\right]+\left[\frac{d Y_{i}}{d t}-\frac{d Y_{i}}{d t}\left(\frac{Y_{i i}}{Y_{i}}\right)\right]\right\} \\
=\bar{l}_{1}\left[\Lambda_{1}-\mu_{1} X_{s}-\frac{X_{s i}}{X_{s}} \Lambda_{1}+\beta_{1} X_{s i} Y_{i}+\mu_{1} X_{s i}-m_{1} X_{i}\right. \\
\left.-\beta_{1} X_{s} Y_{i} \frac{X_{i i}}{X_{r}}+m_{1} X_{i i}+\sigma X_{i}\left(1-\frac{X_{s i}}{X_{s}}\right)\right] \\
+\bar{l}_{2}\left[\Lambda_{2}-\mu_{2} Y_{s}-\frac{Y_{s i}}{Y_{s}} \Lambda_{2}+\beta_{2} Y_{s i} X_{i}+\mu_{2} Y_{s i}-m_{3} Y_{i}\right. \\
\left.\quad-\beta_{2} Y_{s} X_{i} \frac{Y_{i i}}{Y_{i}}+m_{3} Y_{i i}\right] .
\end{aligned}
$$

Substituting $\Lambda_{1}=\beta_{1} X_{s i} Y_{i i}+\mu_{1} X_{s i}-\sigma X_{i i}, \Lambda_{2}=\beta_{2} Y_{s i} X_{i i}+$ $\mu_{2} Y_{s i}, m_{1} X_{i i}=\beta_{1} X_{s i} Y_{i i}$, and $m_{3} Y_{i i}=\beta_{2} Y_{s i} X_{i i}$ into $d L_{2} / d t$, we obtain

$$
\begin{aligned}
\frac{d L_{2}}{d t}=\bar{l}_{1}\left\{\mu_{1} X_{s i}\left(2-\frac{X_{s}}{X_{s i}}-\frac{X_{s i}}{X_{s}}\right)+\left(\beta_{1} X_{s i} Y_{i}-m_{1} X_{i}\right)\right. \\
+\sigma\left(X_{i}-X_{i i}\right)\left(1-\frac{X_{s i}}{X_{s}}\right) \\
\left.+\left[2 \beta_{1} X_{s i} Y_{i i}-\beta_{1} Y_{i i} \frac{\left(X_{s i}\right)^{2}}{X_{s}}-\beta_{1} X_{s} Y_{i} \frac{X_{i i}}{X_{i}}\right]\right\} \\
+\bar{l}_{2}\left\{\mu_{2} Y_{s i}\left(2-\frac{Y_{s}}{Y_{s i}}-\frac{Y_{s i}}{Y_{s}}\right)+\left(\beta_{2} Y_{s i} X_{i}-m_{3} Y_{i}\right)\right. \\
\left.+\left[2 \beta_{2} Y_{s i} X_{i i}-\beta_{2} X_{i i} \frac{\left(Y_{s i}\right)^{2}}{Y_{s}}-\beta_{2} Y_{s} X_{i} \frac{Y_{i i}}{Y_{i}}\right]\right\} .
\end{aligned}
$$

Note that for the limiting system $X_{s}+X_{i}=X_{s i}+X_{i i}=$ $\Lambda_{1} / \mu_{1}$, then $\left(X_{i}-X_{i i}\right)\left(X_{s}-X_{s i}\right) \leq 0$. Let $\bar{l}_{1}=\beta_{2} Y_{s i} X_{i i}$ and $\bar{l}_{2}=\beta_{1} X_{s i} Y_{i i}$. Following from $m_{1} X_{i i}=\beta_{1} X_{s i} Y_{i i}$ and $m_{3} Y_{i i}=$ $\beta_{2} Y_{s i} X_{i i}$, we have

$$
\bar{l}_{1}\left(\beta_{1} X_{s i} Y_{i}-m_{1} X_{i}\right)+\bar{l}_{2}\left(\beta_{2} Y_{s i} X_{i}-m_{3} Y_{i}\right)=0 .
$$


Hence, for all $\left(X_{s}, X_{i}, Y_{s}, Y_{i}\right) \in \Omega_{2}$,

$$
\begin{aligned}
& \frac{d L_{2}}{d t} \leq \bar{l}_{1}\left\{\mu_{1} X_{s i}\left(2-\frac{X_{s}}{X_{s i}}-\frac{X_{s i}}{X_{s}}\right)\right. \\
& \left.+\beta_{1} X_{s i} Y_{i i}\left[2-\frac{X_{s i}}{X_{s}}-\frac{X_{s} Y_{i} X_{i i}}{X_{s i} Y_{i i} X_{i}}\right]\right\} \\
& +\bar{l}_{2}\left\{\mu_{2} Y_{s i}\left(2-\frac{Y_{s}}{Y_{s i}}-\frac{Y_{s i}}{Y_{s}}\right)\right. \\
& \left.\quad+\beta_{2} Y_{s i} X_{i i}\left[2-\frac{Y_{s i}}{Y_{s}}-\frac{Y_{s} X_{i} Y_{i i}}{Y_{s i} X_{i i} Y_{i}}\right]\right\} \\
& \leq \bar{l}_{1} \mu_{1} X_{s i}\left(2-\frac{X_{s}}{X_{s i}}-\frac{X_{s i}}{X_{s}}\right)+\bar{l}_{2} \mu_{2} Y_{s i}\left(2-\frac{Y_{s}}{Y_{s i}}-\frac{Y_{s i}}{Y_{s}}\right) \\
& +\bar{l}_{1} \bar{l}_{2}\left(4-\frac{X_{s i}}{X_{s}}-\frac{X_{s} Y_{i} X_{i i}}{X_{s i} Y_{i i} X_{i}}-\frac{Y_{s i}}{Y_{s}}-\frac{Y_{s} X_{i} Y_{i i}}{Y_{s i} X_{i i} Y_{i}}\right) \leq 0 .
\end{aligned}
$$

It is easy to see that

$$
\begin{gathered}
\frac{d L_{2}}{d t}=0 \Longleftrightarrow X_{s}=X_{s i}, \quad X_{i}=X_{i i} \\
Y_{s}=Y_{s i}, \quad Y_{i}=Y_{i i} .
\end{gathered}
$$

Therefore, the only compact invariant subset of the set where $d L_{2} / d t=0$ is the point $\left(X_{s i}, X_{i i}, Y_{s i}, Y_{i i}\right)$, corresponding to the boundary equilibrium $E_{0 i}$. By LaSalle's Invariance Principle, $E_{0 i}$ is globally asymptotically stable if $R_{0 i}>1$ and $R_{0 r} \leq 1$. Summering above analysis, we have the following result.

Theorem 9. When $\rho=0$, the boundary equilibrium $E_{0 i}$ of the system (1) is globally asymptotically stable if $R_{0 i}>1 \geq R_{0 r}$.

\section{Stability Analysis of the Coexistence Equilibrium}

In this section, we turn to study the local stability of the coexistence equilibrium $E_{c}$ in the limiting system of (1) by using Krasnoselskii sublinearity trick [24], as in $[25,26]$. In detail, if $x^{\prime}=f(x)$ is a system of differential equations and $x^{*}$ is an equilibrium point, then to prove the local asymptotical stability of $x^{*}$ is to prove that the linearized equation $Z^{\prime}=$ $f^{\prime}\left(x^{*}\right) Z$ has no solutions of the form

$$
Z(t)=Z_{0} e^{\omega t}
$$

with $Z_{0} \in C^{n}, \omega \in C$ and, $\operatorname{Re} \omega \geq 0$. This implies that the eigenvalues of the characteristic polynomial associated with the linearized equations have negative real part, that is, $\operatorname{Re} \omega<0$. Then, the coexistence equilibrium $E_{c}$ is locally asymptotically stable.
Considering the limiting system

$$
\begin{aligned}
\frac{d X_{i}}{d t} & =\beta_{1}\left(N_{1}-X_{i}-X_{r}\right) Y_{i}-m_{1} X_{i}, \\
\frac{d X_{r}}{d t} & =k \beta_{1}\left(N_{1}-X_{i}-X_{r}\right) Y_{r}+\rho \sigma X_{i}-m_{2} X_{r}, \\
\frac{d Y_{i}}{d t} & =\beta_{2}\left(N_{2}-Y_{i}-Y_{r}\right) X_{i}-m_{3} Y_{i}, \\
\frac{d Y_{r}}{d t} & =k \beta_{2}\left(N_{2}-Y_{i}-Y_{r}\right) X_{r}-m_{4} Y_{r},
\end{aligned}
$$

where $N_{1}=\Lambda_{1} / \mu_{1}$ and $N_{2}=\Lambda_{2} / \mu_{2}$. In this way, let $Z_{0}=\left(Z_{1}, Z_{2}, Z_{3}, Z_{4}\right), Z_{i} \in C$. Substituting a solution of the form (60) into the linearized system (61) of the coexistence equilibrium $E_{c}$, we obtain the following linear equations:

$$
\begin{aligned}
\omega Z_{1}= & -\left(\beta_{1} Y_{i c}+m_{1}\right) Z_{1}-\beta_{1} Y_{i c} Z_{2} \\
& +\beta_{1}\left(N_{1}-X_{i c}-X_{r c}\right) Z_{3}, \\
\omega Z_{2}= & \left(\rho \sigma-k \beta_{1} Y_{r c}\right) Z_{1}-\left(k \beta_{1} Y_{r c}+m_{2}\right) Z_{2} \\
& +k \beta_{1}\left(N_{1}-X_{i c}-X_{r c}\right) Z_{4}, \\
\omega Z_{3}= & \beta_{2}\left(N_{2}-Y_{i c}-Y_{r c}\right) Z_{1}-\left(\beta_{2} X_{i c}+m_{3}\right)-\beta_{2} X_{i c} Z_{4}, \\
\omega Z_{4}= & k \beta_{2}\left(N_{2}-Y_{i c}-Y_{r c}\right) Z_{2}-k \beta_{2} X_{r c} Z_{3} \\
& -\left(k \beta_{2} X_{r c}+m_{4}\right) Z_{4},
\end{aligned}
$$

which is equivalent to the system

$$
\begin{aligned}
{\left[1+\frac{1}{m_{1}}\left(\omega+\beta_{1} Y_{i c}\right)\right] Z_{1}=} & -\frac{\beta_{1} Y_{i c} Z_{2}}{m_{1}} \\
& +\frac{\beta_{1}\left(N_{1}-X_{i c}-X_{r c}\right)}{m_{1}} Z_{3}, \\
{\left[1+\frac{1}{m_{2}}\left(\omega+k \beta_{1} Y_{r c}\right)\right] Z_{2}=} & \frac{\left(\rho \sigma-k \beta_{1} Y_{r c}\right)}{m_{2}} Z_{1} \\
& +\frac{k \beta_{1}\left(N_{1}-X_{i c}-X_{r c}\right)}{m_{2}} Z_{4} \\
{\left[1+\frac{1}{m_{3}}\left(\omega+\beta_{2} X_{i c}\right)\right] Z_{3}=} & \frac{\beta_{2}\left(N_{2}-Y_{i c}-Y_{r c}\right)}{m_{3}} Z_{1} \\
& -\frac{\beta_{2} X_{i c} Z_{4},}{m_{3}} \\
{\left[1+\frac{1}{m_{4}}\left(\omega+k \beta_{2} X_{r c}\right)\right] Z_{4}=} & \frac{k \beta_{2}\left(N_{2}-Y_{i c}-Y_{r c}\right)}{m_{4}} Z_{2} \\
& -\frac{k \beta_{2} X_{r c}}{m_{4}} Z_{3} .
\end{aligned}
$$


Moving all the negative terms to the left-hand side, after some manipulations we obtain the system

$$
\begin{aligned}
& {\left[1+F_{1}(\omega)\right] Z_{1}+\left[1+F_{2}(\omega)\right] Z_{2}=(H Z)_{1}+(H Z)_{2},} \\
& {\left[1+F_{3}(\omega)\right] Z_{3}+\left[1+F_{4}(\omega)\right] Z_{4}=(H Z)_{3}+(H Z)_{4},}
\end{aligned}
$$

where

$$
\begin{aligned}
& F_{1}(\omega)=\frac{\omega+\beta_{1} Y_{i c}}{m_{1}}+\frac{k \beta_{1} Y_{r c}}{m_{2}}, \\
& F_{2}(\omega)=\frac{\omega+k \beta_{1} Y_{r c}}{m_{2}}+\frac{\beta_{1} Y_{i c}}{m_{1}}, \\
& F_{3}(\omega)=\frac{\omega+\beta_{2} X_{i c}}{m_{3}}+\frac{k \beta_{2} X_{r c}}{m_{4}}, \\
& F_{4}(\omega)=\frac{\omega+k \beta_{2} X_{r c}}{m_{4}}+\frac{\beta_{2} X_{i c}}{m_{3}}, \\
& H=\left(\begin{array}{cccc}
0 & 0 & \frac{\beta_{1}\left(N_{1}-X_{i c}-X_{r c}\right)}{m_{1}} & 0 \\
\frac{\rho \sigma}{m_{2}} & 0 & 0 & k \beta_{1}\left(N_{1}-X_{i c}-X_{r c}\right) \\
\frac{\beta_{2}\left(N_{2}-Y_{i c}-Y_{r c}\right)}{m_{3}} & 0 & 0 & 0 \\
0 & 0 & 0 & \frac{k \beta_{2}\left(N_{2}-Y_{i c}-Y_{r c}\right)}{m_{4}}
\end{array}\right) .
\end{aligned}
$$

Note that the matrix $H$ has nonnegative entries, and $E_{c}=$ $\left(X_{i c}, X_{r c}, Y_{i c}, Y_{r c}\right)$ satisfies

$$
E_{c}=H E_{c} .
$$

To show that Re $\omega<0$, we distinguish two cases: $\omega=0$ and $\omega \neq 0$. In the first case, (62) is a homogeneous linear system. Through calculations, we have the determinant of (62) is

$$
\begin{aligned}
\Delta & =\left|\begin{array}{cccc}
-\left(\beta_{1} Y_{i c}+m_{1}\right) & -\beta_{1} Y_{i c} & \beta_{1} X_{s c} & 0 \\
\rho \sigma-k \beta_{1} Y_{r c} & -\left(k \beta_{1} Y_{r c}+m_{2}\right) & 0 & k \beta_{1} X_{s c} \\
\beta_{2} Y_{s c} & 0 & -\left(\beta_{2} X_{i c}+m_{3}\right) & -\beta_{2} X_{i c} \\
0 & k \beta_{2} Y_{s c} & -k \beta_{2} X_{r c} & -\left(k \beta_{2} X_{r c}+m_{4}\right)
\end{array}\right| \\
& =-\left(\beta_{1} Y_{i c}+m_{1}\right) A_{1}+\beta_{1} Y_{i c} \mathrm{~A}_{2}+\beta_{1} X_{s c} A_{3},
\end{aligned}
$$

where

$$
\begin{aligned}
A_{1}= & m_{1} m_{3} k^{2} \beta_{2} X_{i c}+m_{1} m_{3}^{2} k^{2}-m_{4} k \beta_{1} \beta_{2} X_{i c} Y_{r c} \\
& -m_{3} k^{2} \beta_{1} \beta_{2} X_{r c} Y_{r c}-m_{3} m_{4} k \beta_{1} Y_{r c}-m_{2} m_{4} \beta_{2} X_{i c} \\
& -m_{2} m_{3} k \beta_{2} X_{r c}-m_{2} m_{3} m_{4}, \\
A_{2}= & \rho \sigma\left(m_{3} k \beta_{2} X_{r c}+m_{4} \beta_{2} X_{i c}+m_{3} m_{4}\right)-m_{4} k \beta_{1} \beta_{2} X_{i c} Y_{r c} \\
& -m_{3} k^{2} \beta_{1} \beta_{2} X_{r c} Y_{r c}-m_{3} m_{4} k \beta_{1} Y_{r c}-m_{1} m_{3} k^{2} \beta_{2} X_{r c} \\
A_{3}= & k^{2} \beta_{1} \beta_{2}^{2} X_{s c} Y_{s c}^{2}+\rho \sigma k \beta_{2}^{2} X_{i c} Y_{s c}-k^{2} \beta_{1} \beta_{2}^{2} X_{i c} Y_{r c} Y_{s c} \\
& -\beta_{2} Y_{s c}\left(k^{2} \beta_{1} \beta_{2} X_{r c} Y_{r c}+m_{4} k \beta_{1} Y_{r c}\right. \\
& \left.+m_{2} k \beta_{2} X_{r c}+m_{2} m_{4}\right) .
\end{aligned}
$$

Then, we can obtain

$$
\begin{aligned}
\Delta= & \rho \sigma\left(m_{3} k \beta_{1} \beta_{2} X_{r c} Y_{i c}+m_{4} \beta_{1} \beta_{2} X_{i c} Y_{i c}\right. \\
& \left.\quad+m_{3} m_{4} \beta_{1} Y_{i c}+m_{1} m_{3} k \beta_{2} X_{i c}\right) \\
+ & \left(\beta_{1} \beta_{2} X_{i c} Y_{i c}+m_{1} \beta_{2} X_{i c}+m_{3} \beta_{2} Y_{i c}\right) \\
+ & \left(m_{2} m_{4}-m_{1} m_{3} k^{2}\right)+\beta_{1} \beta_{2} X_{r c} Y_{i c} m_{3} k\left(m_{2}-m_{1} k\right) \\
+ & \beta_{1} \beta_{2} X_{i c} Y_{r c} m_{1} k\left(m_{4}-m_{3} k\right) .
\end{aligned}
$$

Note that the coexistence equilibrium $E_{c}$ exists under the condition $R_{0 i}>R_{0 r}$, that is, $m_{2} m_{4}>m_{1} m_{3} k^{2}$. Then $\Delta>0$ if $m_{2}-m_{1} k \geq 0$ and $m_{4}-m_{3} k \geq 0$. Hence, for the case that $\omega=0$, (62) has only the trivial solution if $m_{2}-m_{1} k \geq 0$ and $m_{4}-m_{3} k \geq 0$. This implies that $\omega \neq 0$.

Now assume that $\omega \neq 0$ and $\operatorname{Re} \omega \geq 0$. Let $F(\omega)=\min \{\mid 1+$ $\left.F_{i}(\omega) \mid, i=1,2,3,4\right\}$. It is easy to prove that $\left|1+F_{i}(\omega)\right|>1$ for all $i$, and therefore $F(\omega)>1$. In the other hand, since the coordinates of $E_{c}$ are positive, if $\bar{Z}$ is any solution of (64), then there exists a minimal positive real number $s$ such that

$$
|\bar{Z}| \leq s E_{c}
$$

where $|\bar{Z}|=\left(\left|Z_{1}\right|,\left|Z_{2}\right|,\left|Z_{3}\right|,\left|Z_{4}\right|\right)$, and $|\cdot|$ is the norm in $C$. Note that $s$ is also the minimal positive real number such that

$$
\left|Z_{1}\right|+\left|Z_{2}\right| \leq s\left(X_{i c}+X_{r c}\right), \quad\left|Z_{3}\right|+\left|Z_{4}\right| \leq s\left(Y_{i c}+Y_{r c}\right) .
$$

Taking norms on both side of (64) and using (66), (71), and the fact that $H$ is nonnegative, we obtain

$$
F(\omega)\left(\left|Z_{1}\right|+\left|Z_{2}\right|\right) \leq H(|Z|)_{1}+H(|Z|)_{2} \leq s\left(X_{i c}+X_{r c}\right),
$$

that is,

$$
\left|Z_{1}\right|+\left|Z_{2}\right| \leq \frac{s}{F(\omega)}\left(X_{i c}+X_{r c}\right)<s\left(X_{i c}+X_{r c}\right),
$$

which contradicts the minimality of $s$. Hence, $\operatorname{Re} \omega<0$.

Summering above analysis, we have the following result.

Theorem 10. When $\rho \neq 0$, the coexistence equilibrium $E_{c}$ of the system (1) is locally asymptotically stable if $k \leq$ $\min \left\{m_{2} / m_{1}, m_{4} / m_{3}\right\}$ and $R_{0 i}>1$.

The existence and stability of equilibria can be summered in Table 1.

\section{Discussion}

In this paper we established a new schistosomiasis model. In contrast to previous schistosomiasis models with drug resistance, the model established in this study consider many aspects. First, snail is considered as a variable in the model since the resistance of schistosoma to PZQ can be expressed in snails [14-16]. Second, previous models considered that 
TABLE 1: Existence and stability of equilibria. The sign “ $\exists$ !” means the existence and uniqueness. LAS means locally asymptotically stable, and GAS means globally asymptotically stable.

\begin{tabular}{lllll}
\hline & & & & $\rho \neq 0$ \\
& Existence & Stability & Existence & Stability \\
\hline$E_{0}$ & $\exists !$ & $\begin{array}{l}\text { If } R_{0}<1, \mathrm{LAS} ; \\
\text { If } R_{0} \leq 1, \mathrm{GAS}\end{array}$ & $\exists !$ & $\begin{array}{l}\text { If } R_{0}<1, \mathrm{LAS} ; \\
\text { If } R_{0} \leq 1, \mathrm{GAS}\end{array}$ \\
\hline$E_{0 i}$ & If $R_{0 i}>1, \exists !$ & $\begin{array}{l}\text { If } R_{0 i}>1 \text { and } R_{0 i}>R_{0 r}, \mathrm{LAS} ; \\
\text { If } R_{0 i}>1 \geq R_{0 r}, \mathrm{GAS}\end{array}$ & Not exist & $/$ \\
\hline$E_{0 r}$ & If $R_{0 r}>1, \exists !$ & $\begin{array}{l}\text { If } R_{0 r}>1 \text { and } R_{0 r}>R_{0 i}, \text { LAS; } \\
\text { If } R_{0 r}>1 \geq R_{0 i}, \mathrm{GAS}\end{array}$ & If $R_{0 r}>1, \exists !$ & $\begin{array}{l}\text { If } R_{0 r}>1 \text { and } R_{0 r}>R_{0 i}, \text { LAS; } \\
\text { If } R_{0 r}>1 \geq R_{0 i}, \mathrm{GAS}\end{array}$ \\
\hline$E_{c}$ & $\begin{array}{l}\text { If } R_{0 i}=R_{0 r}>1, \\
\text { a line }\end{array}$ & $/$ & If $R_{0 i}>1$ and $R_{0 i}>R_{0 r}, \exists !$ & $\begin{array}{l}\text { If } R_{0 i}>1 \text { and } \\
k \leq \text { min }\left\{m_{2} / m_{1}, m_{4} / m_{3}\right\}, \text { LAS }\end{array}$
\end{tabular}

resistance was caused by drug treatment, while a large number of the literature show that some cases are due to inheritance $[1,3,4]$. Therefore, in this study, we consider the reasons for resistance are drug treatment and genetic. Last, in previous models a resistance level $(\theta)$ was used to discount the treatment rate. But the resistance level could not be measured, and its value was assumed. This model consider what percentage of infected human after treatment will recover and what percentage will emerge drug resistance. In medicine, from the occurrence of cases the value of this ratio can be identified. For example, this ratio was given in $[27,28](1-\rho=0.28 \sim$ $0.609)$. Therefore, it is easy to operate. In addition, we separate the discussion of the case that treatment will cause resistance $(\rho \neq 0)$ and treatment will not cause resistance $(\rho=0)$.

The reproductive number $R_{0 i}$ and $R_{0 r}$ of the sensitive and resistant strains are given, respectively. It is easy to see that $R_{0 i}$ is a decreasing function of $\sigma$, and $R_{0 r}$ is an increasing function of $k$. If the basic reproductive number $R_{0}=\max \left\{R_{0 i}, R_{0 r}\right\}$ of the model (1) is less than 1 , one can prove the stability of the disease free equilibrium. This means that the spread of schistosomiasis can be effectively controlled. When the basic reproductive number $R_{0}$ is greater than 1 , we first consider the case that $\rho=0$. If $R_{0 i}=R_{0 r}>1$, the two strains can coexist. There is a line of coexistence equilibria in this case. The infected human will evolve to one of them with higher reproductive number (see Table 1 ).

When drug treatment can not cause resistance $(\rho=0)$, that is, the new resistant strain is due to the inheritance of resistance, the sensitive strain will dominate if the treatment rate is smaller and the impact of the inheritance of resistance is smaller such that $R_{0 i}>1 \geq R_{0 r}$. This result accords to the results of previous models. On the other hand, if the treatment rate and the impact of the inheritance of resistance are both larger such that $R_{0 r}>1 \geq R_{0 i}$, the resistant strain will dominate. This shows that although the assumption is that drug treatment does not result in the emergence of drug resistance, once the treatment rate is greater than a value, and the impact of genetic resistance is larger, there will still be the emergence of resistant strain, and the resistant strain is dominant. This further shows that genetic resistance has a great impact on the system.

When drug treatment can cause resistance $(\rho \neq 0)$, we can show that the sensitive strain either does not appear or coexist with the resistant strain under certain condition. If $R_{0 r}>1$, there is only resistant strain. It can be seen that, regardless of whether drug treatment leads to the emergence of resistance, once the impact of genetic resistance is larger, resistant strain will be dominant, which is detrimental to the control of schistosomiasis.

Finally, from the formula of the coexistence equilibrium $E_{c}$, it is easy to see that the value of the resistant strain is increased with the value of $\rho$. This means once the proportion of human with drug-resistant strain produced by drug treatment is larger, the number of human and snails with resistant strain is larger. This is not a good result for drug treatment with praziquantel.

Hence, for poor treatment, there are two possible reasons: drug therapy and genetic. An important priority in developing new control strategies is to search new drug targets, in combination with selection of genetic methods such as that viable vaccine candidates. And there is already a need for alternative drugs to treat PZQ-resistant schistosomiasis, such as already exists in northern Senegal [10].

\section{Acknowledgments}

This paper is supported by National Natural Science Foundation of China (11126177 and 11071011), Natural Science Foundation of Anhui Province (1208085QA15), and by the Foundation for Young Talents in College of Anhui Province (2012SQRL021) and also supported by Doctoral Fund of Ministry of Education of China (20113401110001 and 20103401120002), the Key Natural Science Foundation of the Anhui Higher Education Institutions of China (KJ2009A49), and Funding Project for Academic Human Resources Development in Institutions of Higher Learning Under the Jurisdiction of Beijing Municipality (PHR201107123). The authors would like to thank anonymous reviewers for very helpful suggestions which improved greatly this paper.

\section{References}

[1] D. Cioli, "Chemotherapy of schistosomiasis: an update," Parasitology Today, vol. 14, no. 10, pp. 418-422, 1998.

[2] P. G. Fallon, L. F. Tao, M. M. Ismail, and J. L. Bennett, "Schistosome resistance to praziquantel: fact or artifact?" Parasitology Today, vol. 12, no. 8, pp. 316-320, 1996. 
[3] D. Cioli, "Praziquantel: is there real resistance and are there alternatives?" Current Opinion in Infectious Diseases, vol. 13, no. 6, pp. 659-663, 2000.

[4] M. Ismail, S. Botros, A. Metwally et al., "Resistance to praziquantel: direct evidence from Schistosoma mansoni isolated from egyptian villagers," American Journal of Tropical Medicine and Hygiene, vol. 60, no. 6, pp. 932-935, 1999.

[5] B. Gryseels, F. F. Stelma, I. Talla et al., "Epidemiology, immunology and chemotherapy of Schistosoma mansoni infections in a recently exposed community in Senegal," Tropical and Geographical Medicine, vol. 46, no. 4, pp. 209-219, 1994.

[6] F. F. Stelma, I. Talla, S. Sow et al., "Efficacy and side effects of praziquantel in an epidemic focus of Schistosoma mansoni," American Journal of Tropical Medicine and Hygiene, vol. 53, no. 2, pp. 167-170, 1995.

[7] P. G. Fallon, R. F. Sturrock, A. Capron, M. Niang, and M. J. Doenhoff, "Short report: diminished susceptibility to praziquantel in a Senegal isolate of Schistosoma mansoni," American Journal of Tropical Medicine and Hygiene, vol. 53, no. 1, pp. 6162, 1995.

[8] Y. S. Liang, G. C. Coles, M. J. Doenhoff, and V. R. Southgate, "In vitro responses of praziquantel-resistant and -susceptible Schistosoma mansoni to praziquantel," International Journal for Parasitology, vol. 31, no. 11, pp. 1227-1235, 2001.

[9] M. Ismail, A. Metwally, A. Farghaly, J. Bruce, L. F. Tao, and J. L. Bennett, "Characterization of isolates of Schistosoma mansoni from Egyptian villagers that tolerate high doses of praziquantel," American Journal of Tropical Medicine and Hygiene, vol. 55, no. 2, pp. 214-218, 1996.

[10] M. J. Doenhoff, J. R. Kusel, G. C. Coles, and D. Cioli, "Resistance of Schistosoma mansoni to praziquantel: is there a problem?" Transactions of the Royal Society of Tropical Medicine and Hygiene, vol. 96, no. 5, pp. 465-469, 2002.

[11] C. Castillo-Chavez, Z. Feng, and D. Xu, "A schistosomiasis model with mating structure and time delay," Mathematical Biosciences, vol. 211, no. 2, pp. 333-341, 2008.

[12] Z. Feng, J. Curtis, and D. J. Minchella, "The influence of drug treatment on the maintenance of schistosome genetic diversity," Journal of Mathematical Biology, vol. 43, no. 1, pp. 52-68, 2001.

[13] D. Xu, J. Curtis, Z. Feng, and D. J. Minchella, "On the role of schistosome mating structure in the maintenance of drug resistant strains," Bulletin of Mathematical Biology, vol. 67, no. 6, pp. 1207-1226, 2005.

[14] Y. S. Liang, J. R. Dai, Y. C. Zhu, G. C. Coles, and M. J. Doenhoff, "Genetic analysis of praziquantel resistance in Schistosoma mansoni," Southeast Asian Journal of Tropical Medicine and Public Health, vol. 34, no. 2, pp. 274-280, 2003.

[15] Y. S. Liang, J. R. Dai, Y. C. Zhu et al., "Studies on resistance of schistosoma to praziquante IIX. Genetic analysis of praziquantel resistance in Schistosoma mansoni," Chinese Journal of Schistosomiasis Control, vol. 16, pp. 81-85, 2004.

[16] W. Wang and Y. S. Liang, "Progress on research of resistance of schistosome to praziquantel," International Journal of Medical Parasitic Diseases, vol. 34, pp. 291-296, 2007 (Chinese).

[17] Z. Feng, C. C. Li, and F. A. Milner, "Schistosomiasis models with density dependence and age of infection in snail dynamics," Mathematical Biosciences, vol. 177-178, pp. 271-286, 2002.

[18] Z. Feng, C. C. Li, and F. A. Milner, "Schistosomiasis models with two migrating human groups," Mathematical and Computer Modelling, vol. 41, no. 11-12, pp. 1213-1230, 2005.
[19] P. Zhang, G. J. Sandland, Z. Feng, D. Xu, and D. J. Minchella, "Evolutionary implications for interactions between multiple strains of host and parasite," Journal of Theoretical Biology, vol. 248, no. 2, pp. 225-240, 2007.

[20] E. J. Allen and H. D. Victory Jr., "Modelling and simulation of a schistosomiasis infection with biological control," Acta Tropica, vol. 87, no. 2, pp. 251-267, 2003.

[21] O. Diekmann and J. A. P. Heesterbeek, Mathematical Epidemiology of Infectious Diseases: Model Building, Analysis and Interpretation, Wiley Series in Mathematical and Computational Biology, John Wiley \& Sons, New York, NY, USA, 2000.

[22] P. van den Driessche and J. Watmough, "Reproduction numbers and sub-threshold endemic equilibria for compartmental models of disease transmission," Mathematical Biosciences, vol. 180, pp. 29-48, 2002.

[23] J. C. Kamgang and G. Sallet, "Computation of threshold conditions for epidemiological models and global stability of the disease-free equilibrium (DFE)," Mathematical Biosciences, vol. 213, no. 1, pp. 1-12, 2008.

[24] M. A. Krasnosel'skiŭ, Positive Solutions of Operator Equations, P. Noordhoff, Groningen, The Netherlands, 1964.

[25] L. Esteva and C. Vargas, "Influence of vertical and mechanical transmission on the dynamics of dengue disease," Mathematical Biosciences, vol. 167, no. 1, pp. 51-64, 2000.

[26] H. W. Hethcote and H. R. Thieme, "Stability of the endemic equilibrium in epidemic models with subpopulations," Mathematical Biosciences, vol. 75, no. 2, pp. 205-227, 1985.

[27] G. Raso, E. K. N’Goran, A. Toty et al., "Efficacy and side effects of praziquantel against Schistosoma mansoni in a community of western Côte d'Ivoire," Transactions of the Royal Society of Tropical Medicine and Hygiene, vol. 98, no. 1, pp. 18-27, 2004.

[28] L. van Lieshout, F. F. Stelma, F. Guissé et al., "The contribution of host-related factors to low cure rates of praziquantel for the treatment of Schistosoma mansoni in Senegal," American Journal of Tropical Medicine and Hygiene, vol. 61, no. 5, pp. 760-765, 1999. 


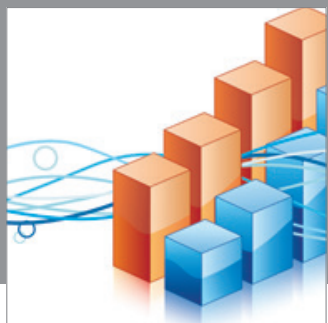

Advances in

Operations Research

mansans

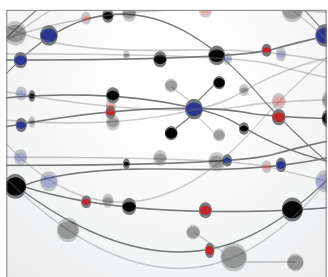

The Scientific World Journal
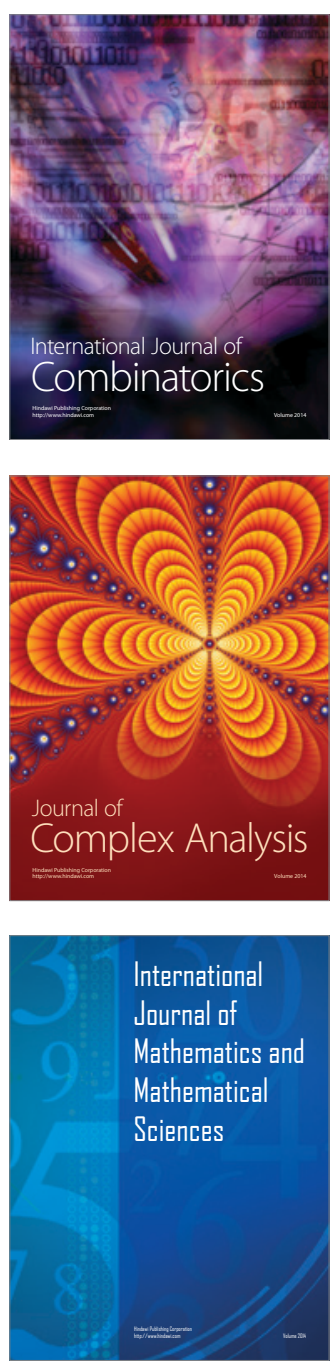
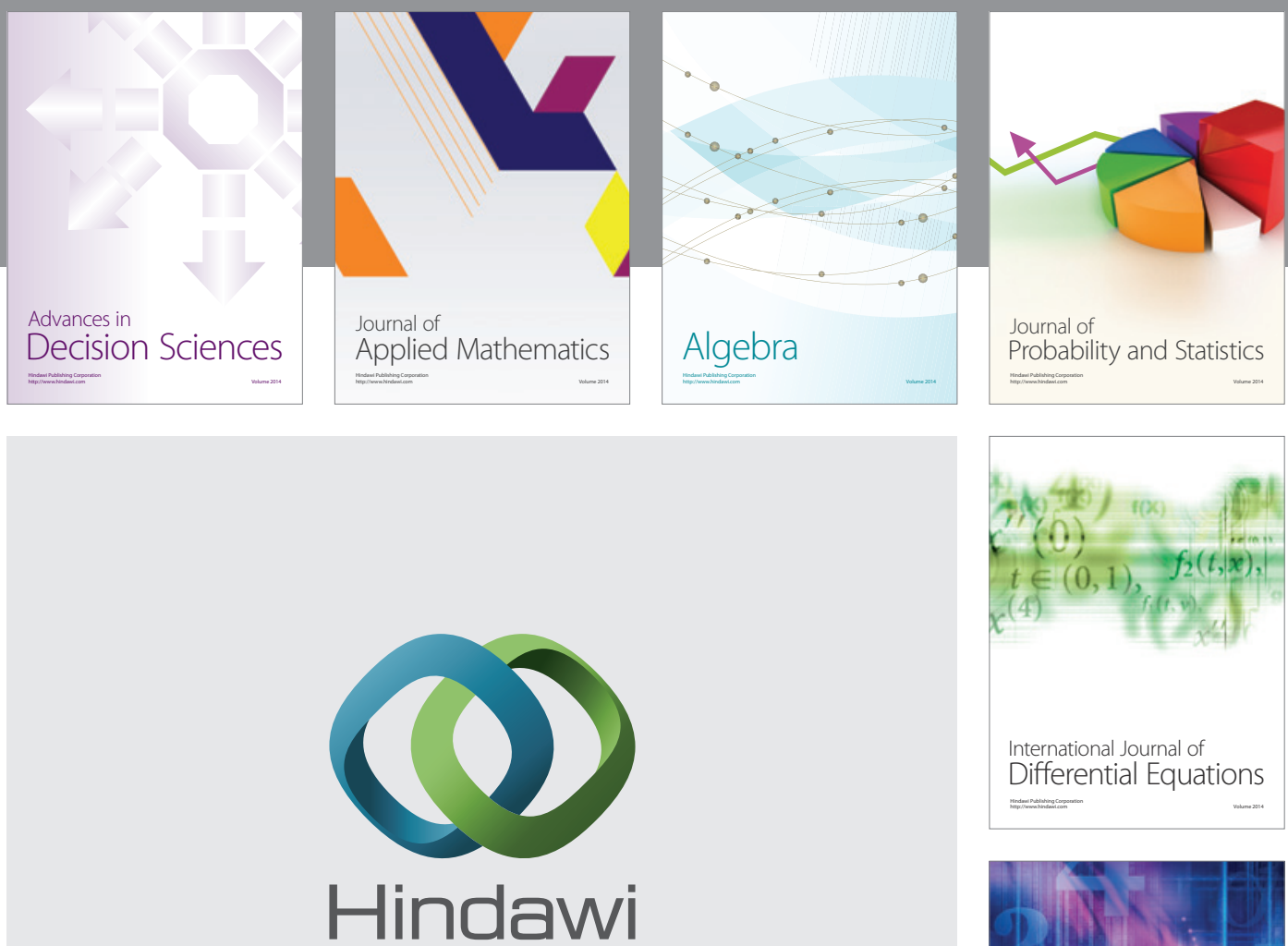

Submit your manuscripts at http://www.hindawi.com
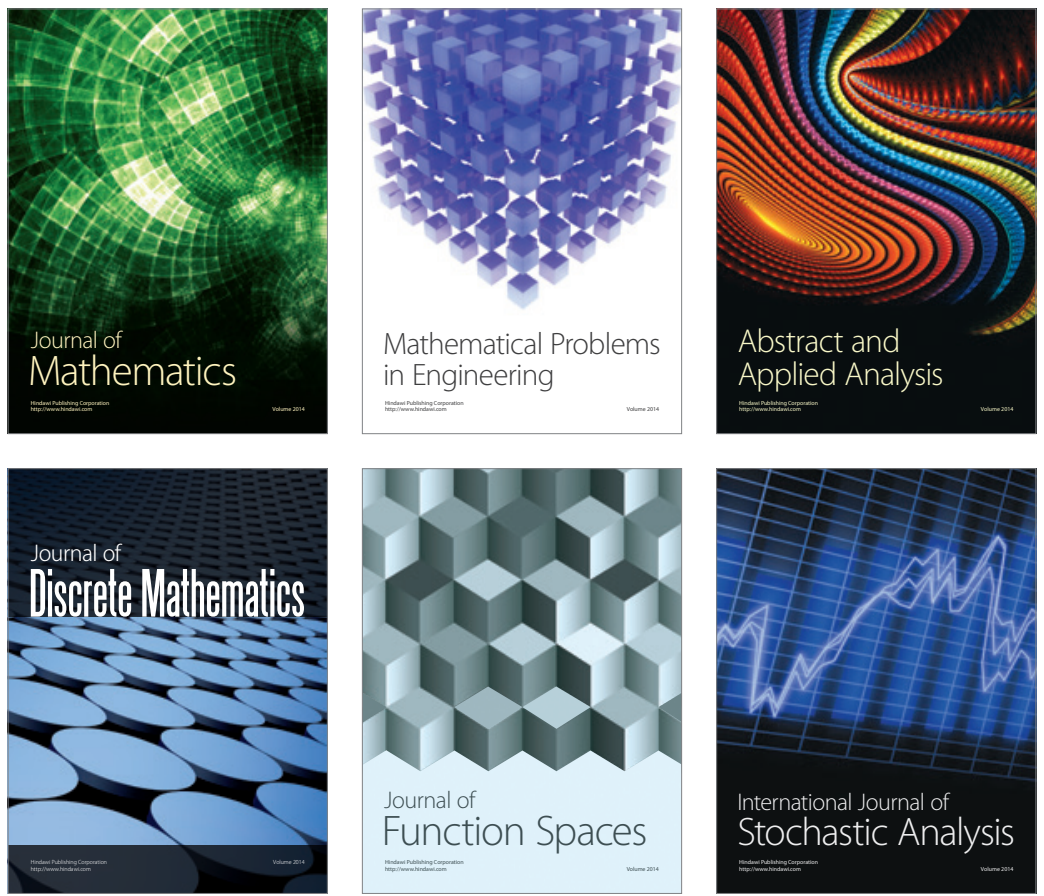

Journal of

Function Spaces

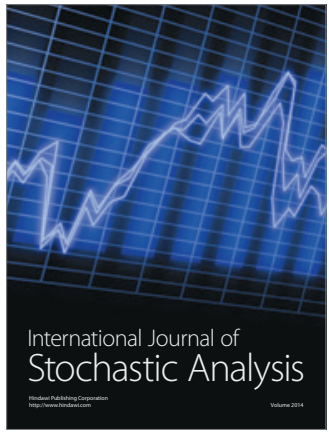

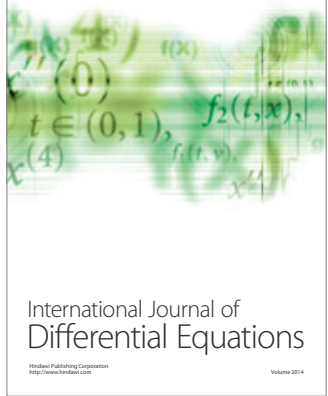
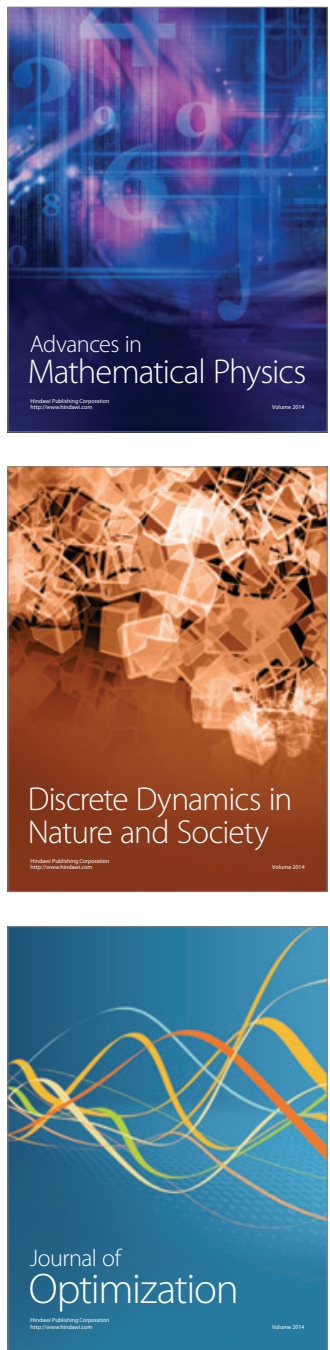